\title{
Long-time behavior and Turing instability induced by cross-diffusion in a three species food chain model with a Holling type-II functional response
}

\author{
Dawit Haile and Zhifu Xie \\ Department of Mathematics and Economics \\ Virginia State University \\ Petersburg, Virginia 23806, USA \\ Email: zxie@vsu.edu
}

\begin{abstract}
In this paper, we study a strongly coupled reaction-diffusion system describing three interacting species in a food chain model, where the third species preys on the second one and simultaneously the second species preys on the first one. An intra-species competition $b_{2}$ among the second predator is introduced to the food chain model. This parameter produce some very interesting result in linear stability and Turing instability. We first show that the unique positive equilibrium solution is locally asymptotically stable for the corresponding ODE system when the intra-species competition exists among the second predator. The positive equilibrium solution remains linearly stable for the reaction diffusion system without cross diffusion, hence it does not belong to the classical Turing instability scheme. But it becomes linearly unstable only when cross-diffusion also plays a role in the reaction-diffusion system, hence the instability is driven solely from the effect of cross diffusion. Our results also exhibit some interesting combining effects of cross-diffusion, intra-species competitions and inter-species interactions. Numerically, we conduct a one parameter analysis which illustrate how the interactions change the existence of stable equilibrium, limit cycle, and chaos. Some interesting dynamical phenomena occur when we perform analysis of interactions in terms of selfproduction of prey and intra-species competition of the middle predator. By numerical simulations, it illustrates the existence of nonuniform steady solutions and new patterns such as spot patterns, strip patterns and fluctuations due to the diffusion and cross diffusion in 2-dimension.
\end{abstract}

Key word: Predator-prey models; Reaction-diffusion system; Cross-diffusions; Turing instability; Turing patterns; Food chain.

AMS classification number 35K57, 35B36, 37D45, 92C15, 92D40. 


\section{Introduction}

In population dynamics, the predator-prey systems have been studied and the analysis of food chains is an active research area in the mathematical biological science $[9,10]$. Food chains or food webs are made up of a number of species linked by tropic interactions [1]. A simple food chain consists of linear links in a food web. Here we consider a simple food chain which consists of three species where the third species preys on the second one and simultaneously the second species preys on the first one. There are several interesting cases of simple food chains of three interacting species categorized by the types of predators. It includes the case where there is a generalist predator and a specialist predator. It also includes the case where both predators are generalists or specialists. A predator is a generalist if it can change its food source in the absence of its favorite food which is very common in nature. Chaotic dynamics was observed in a food chain by Hastings and Powell [5] where both the top-predator and the middle-predator are specialists.

Upadhyay, Iyengar, and Rai $[17,18]$ proposed a model to understand the reasons why chaos is rarely observed in natural populations of three interacting species, where the toppredator is a generalist while the middle-predator is a specialist. The model and its variants have been studied by several authors $[1,19]$. Most of these studies concentrate on the original model with different functional response, which is spatially homogeneous. There are few works on pattern formation in a spatially inhomogeneous model of this type.

Spatial spread of species is everywhere in nature. The spread can lead to interesting patterns. The species might disperse in order to find food, seek refuge, find mates etc. Among the various pattern formations, Turing patterns are generated when the species in the food chain have different diffusion coefficients. In [16], Wang studied an elliptic system which models the dynamics of a two-preys-one-predator ecosystem and he showed that the cross-diffusions can create the stationary patterns where cross-diffusions are included in such a way that predator chases the prey and the prey runs away from the predator. In [24], Xie studied a simple food chain model where both predators are generalists. He showed that the positive equilibrium solution for the model is globally asymptotically stable for the reaction diffusion system without cross diffusion by constructing a Lyapunov function, hence it does not belong to the classical Turing instability scheme. But it becomes linearly unstable only when cross-diffusion also plays a role in the reaction-diffusion system, hence the instability is driven solely from the effect of cross diffusion. There are numerous research papers of predator prey model, for example, $[2,3,4,12,27]$ for two species and $[6,8,11,7]$ for three species. We just mention some of them here which are closely related to the current paper and more references can be found therein.

The following simple food chain is modified from the classical Upadhyay-Iyengar-Rai model [17] by adding intra-species competition $\left(b_{2} \neq 0\right)$ in the middle specialist predator. 


$$
\left\{\begin{array}{l}
\frac{d u_{1}}{d t}=u_{1}\left(a_{1}-b_{1} u_{1}-\frac{w_{0} u_{2}}{u_{1}+D_{0}}\right), \\
\frac{d u_{2}}{d t}=u_{2}\left(-a_{2}-b_{2} u_{2}+\frac{w_{1} u_{1}}{u_{1}+D_{1}}-\frac{w_{2} u_{3}}{u_{2}+D_{2}}\right), \\
\frac{d u_{3}}{d t}=u_{3}^{2}\left(a_{3}-\frac{w_{3}}{u_{2}+D_{3}}\right) .
\end{array}\right.
$$

The three species in the model are denoted by $u_{i}, i=1,2,3$. The top generalist predator $u_{3}$ preys on $u_{2}$, which is its favorite food. In turn, the middle specialist predator $u_{2}$ preys on its only food, the prey $u_{1}$. The interaction between the specialist predator $u_{2}$ and its prey $u_{1}$ is modeled by Volterra scheme (predator population dies out exponentially in the absence of its food). The interaction between the specialist predator $u_{2}$ and the generalist predator $u_{3}$ is modeled by the Leslie-Gower scheme. The function response is Holling type II. There are 13 nonnegative parameters in the model and their biological definitions can be found in $[9,10,17] . a_{1}$ is the growth rate of prey $u_{1} . a_{2}$ is the rate at which $u_{2}$ dies out when there is no food $u_{1}$ and no predator $u_{3}$. $a_{3}$ measures the growth rate of $u_{3}$ via sexual reproduction. $b_{1}$ and $b_{2}$ describe the intra-competition among prey $u_{1}$ and among specialist predator $u_{2}$ respectively. $w_{i}$ is the maximum value which per capita reduction rate can attain. $D_{0}$ and $D_{1}$ signifies the extent to which environment provides protection to the prey $u_{1}$. $D_{2}$ is the value of $u_{2}$ at which its per capital removal rate becomes $w_{2} / 2$ and $D_{3}$ represents the residual loss in $u_{3}$ population due to the severe scarcity of its favorite food $u_{2}$. This ODE model has very rich dynamical phenomena. Simulations of this model with $b_{2}=0$ in [17] show the existence of different solutions such as the stable equilibrium, states of extinction, limit cycles, and chaotic behaviors in various ranges of the parameter space.

One of the goals in this paper is to investigate the effects of the intra-species competition among the specialist predators $\left(b_{2} \neq 0\right)$ and the effects of diffusions between species which were not included in the previous papers $[15,17,21]$. It is also to understand the Turing pattern formations due to the spatial interactions in the three species food chain diffusive model.

$$
\left\{\begin{array}{l}
u_{1 t}=\triangle\left[\left(k_{11}+k_{12} u_{2}\right) u_{1}\right]+u_{1}\left(a_{1}-b_{1} u_{1}-\frac{w_{0} u_{2}}{u_{1}+D_{0}}\right), \\
u_{2 t}=\triangle\left[\left(k_{21} u_{1}+k_{22}+k_{23} u_{3}\right) u_{2}\right]+u_{2}\left(-a_{2}-b_{2} u_{2}+\frac{w_{1} u_{1}}{u_{1}+D_{1}}-\frac{w_{2} u_{3}}{u_{2}+D_{2}}\right), \\
u_{3 t}=\triangle\left[\left(k_{32} u_{2}+k_{33}\right) u_{3}\right]+u_{3}^{2}\left(a_{3}-\frac{w_{3}}{u_{2}+D_{3}}\right) \text { in } \Omega \times(0, \infty), \\
\frac{\partial u_{1}}{\partial n}=\frac{\partial u_{2}}{\partial n}=\frac{\partial u_{3}}{\partial n}=0 \text { on } \partial \Omega \times(0, \infty), \\
u_{i}(x, 0)=u_{i 0}(x) \text { in } \Omega \text { for } i=1,2,3,
\end{array}\right.
$$

where $\Omega$ is a bounded domain with smooth boundary in $\mathbf{R}^{N}(N \geq 1)$ and $n$ is the outward unit normal vector on boundary $\partial \Omega$. The homogeneous Neumann boundary condition 
means that the three species have zero flux across the boundary. $k_{i i}$ is the diffusion rate of $i$-th species and $k_{i j}(i \neq j, 1 \leq i, j \leq 3)$ is the cross-diffusion rate of $i$-th species due to the pressure of the presence of $j$-th species. Here $k_{13}$ and $k_{31}$ are missing in (2), which means that the third and first species in the simple food chain do not have direct interaction.

The role of diffusion and cross-diffusion in the modeling of many physical, chemical and biological processes has been extensively studied. A pure diffusion process usually leads to a stabilizing effect so that the system tends to a constant equilibrium state. However in [14], Alan Turing suggested that, under certain conditions, chemicals can react and diffuse in such a way as to produce non-constant equilibrium solutions, where diffusion is actually a destabilizing factor. Diffusion can destablize an initially stable state due to inhomogeneous perturbation. Turing patterns occur when the stable homogeneous steady state of the system is broken.

In [21], Parshad etc. attempted to study the Turing instability for the positive equilibrium for a similar food chain system with self-diffusion only where all $k_{i j}=0$ for $i \neq j$ and intra-species interaction $b_{2}=0$. They proved the existence of a finite dimensional global attractor and they also numerically found some regions where the Turing instability occurs. In [15], Tian studies a very similar model with a different third equation of top species and he presents a rigorous proof with the assumption that their model has at least one nonhomogenous stationary solution by the Leray-Schauder degree theory. Both papers numerically found some interesting Turing patterns due to the self-diffusion or cross-difussion.

In this paper, we will theoretically prove the existence of the Turing instability in the case $k_{i j} \geq 0$. We first find all possible equilibrium solutions to the ODE system (1). Then we conduct Turing instability analysis of the equilibrium solutions to the diffusive system (2). Finally the rich dynamical phenomena of the ODE system is studied for some given parameters. Then we investigate pattern formation and diffusion driven instability via numerical simulations in two dimensions.

The paper is organized as follows. In section 2, we present the equilibrium solutions of the model and we prove that the positive coexistence equilibrium is locally asymptotically stable. In section 3, we perform Turing instability analysis and we prove that Turing instability does not happen if the conditions (7) are satisfied and only self diffusions $\left(k_{i i}>0, k_{i j}=0\right)$ occur. In section 4 , we prove that Turing instability can be induced by cross diffusion. In the last section, numerical simulation is to illustrate the rich dynamical phenomena and the existence of new patterns. 


\section{Stability Analysis of the Equilibriums of the ODE System}

Let $\mathbf{u}=\left(u_{1}, u_{2}, u_{3}\right)^{T}$ be an equilibrium solution of the ODE system (1), i.e. $\frac{d u_{i}}{d t}=0$, $i=1,2,3$. By solving the algebraic system,

$$
\left\{\begin{array}{l}
u_{1} g_{1}\left(u_{1}, u_{2}, u_{3}\right):=u_{1}\left(a_{1}-b_{1} u_{1}-\frac{w_{0} u_{2}}{u_{1}+D_{0}}\right)=0 \\
u_{2} g_{2}\left(u_{1}, u_{2}, u_{3}\right):=u_{2}\left(-a_{2}-b_{2} u_{2}+\frac{w_{1} u_{1}}{u_{1}+D_{1}}-\frac{w_{2} u_{3}}{u_{2}+D_{2}}\right)=0 \\
u_{3}^{2} g_{3}\left(u_{1}, u_{2}, u_{3}\right):=u_{3}^{2}\left(a_{3}-\frac{w_{3}}{u_{2}+D_{3}}\right)=0
\end{array}\right.
$$

we can get the following equilibrium solutions:

$$
\begin{gathered}
\mathbf{u}^{(1)}=(0,0,0) ; \\
\mathbf{u}^{(2)}=\left(\frac{a_{1}}{b_{1}}, 0,0\right) ; \\
\mathbf{u}^{(3)}=\left(0,-\frac{a_{2}}{b_{2}}, 0\right) ; \\
\mathbf{u}^{(4)}=\left(0, \frac{w_{3}-a_{3} D_{3}}{a_{3}},-\frac{\left(a_{2}+b_{2} u_{2}\right)\left(u_{2}+D_{2}\right)}{w_{2}}\right) ;
\end{gathered}
$$

$\mathbf{u}^{(5)}=(0,0, \alpha)$ if $w_{3}=a_{3} D_{3}$, where $\alpha$ is any positive constant;

$\mathbf{u}^{(6)}=\left(\frac{a_{1}}{b_{1}}, 0, \alpha\right)$ if $w_{3}=a_{3} D_{3}$, where $\alpha$ is any positive constant;

$\mathbf{u}^{(7)}=\left(u_{1},\left(a_{1}-b_{1} u_{1}\right)\left(u_{1}+D_{0}\right) / w_{0}, 0\right)$, where $u_{1}$ is a real solution of a third degree polynomial from $g_{2}=0$ after $u_{2}=\left(a_{1}-b_{1} u_{1}\right)\left(u_{1}+D_{0}\right) / w_{0}$ and $u_{3}=0$ are substituted into $g_{2}$.

$$
\mathbf{u}^{(8)}=\left(u_{1}^{-}, u_{2}^{-}, u_{3}^{-}\right) \text {, and }
$$

$$
\mathbf{u}^{(9)}=\left(u_{1}^{+}, u_{2}^{+}, u_{3}^{+}\right), \text {where } u_{2}^{-}=u_{2}^{+}=\frac{w_{3}}{a_{3}}-D_{3} \text { by } g_{3}=0 \text { and }
$$

$u_{1}^{ \pm}$is the two roots by the quadratic formula for the quadratic equation of $u_{1}$ resulted from $g_{1}=0$.

$$
u_{1}^{ \pm}=\frac{\left(a_{1}-b_{1} D_{0}\right) \pm \sqrt{\left(a_{1}+b_{1} D_{0}\right)^{2}-4 b_{1} w_{0} u_{2}^{ \pm}}}{2 b_{1}} .
$$

$u_{3}^{ \pm}$can be solved for the given $u_{1}^{ \pm}$and $u_{2}^{ \pm}$from $g_{2}=0$ and

$$
u_{3}^{ \pm}=\frac{\left(u_{2}^{ \pm}+D_{2}\right)}{w_{2}}\left(-a_{2}-b_{2} u_{2}^{ \pm}+\frac{w_{1} u_{1}^{ \pm}}{u_{1}^{ \pm}+D_{1}}\right) .
$$

All components of $\mathbf{u}$ must be real and nonnegative since they are the population densities of the species. It is easy to see that solutions $\mathbf{u}^{(3)}$ and $\mathbf{u}^{(4)}$ have at least one negative component. Solutions $\mathbf{u}^{(5)}$ and $\mathbf{u}^{(6)}$ are very peculiar because they exist only when $w_{3}=$ $a_{3} D_{3}$. Solutions $\mathbf{u}^{(8)}$ and $\mathbf{u}^{(9)}$ are the only coexistence equilibria and all others have the 
extinction of one or more species. When we conduct numerical simulations to illustrate various Turing patterns, the equilibrium solution $\mathbf{u}^{(8)}$ exhibits negative components for the parameters we used. Thus, the equilibrium solution $\mathbf{u}^{(9)}$ is the best choice for Turing instability analysis from the biological point view.

Roughly speaking, the top generalist predator $u_{3}$ becomes blowup in finite time when $a_{3} \geq \frac{w_{3}}{D_{3}}$ which makes $\frac{d u_{3}}{d t} \geq 0$ for any time $t$. We should note that to make the coexistence equilibrium $\mathbf{u}^{(9)}$ happen, the parameters $a_{3}, w_{3}, D_{3}$ have to be chosen in such a way that $a_{3}<\frac{w_{3}}{D_{3}}$. However, even when $a_{3}<\frac{w_{3}}{D_{3}}$, the top generalist predator can still blowup because it is possible that $\frac{w_{2}}{u_{2}+D_{3}}<a_{3}<\frac{w_{3}}{D_{3}}$ for some values $u_{2}$ of the middle specialist predator. For large enough initial value $u_{3}$, blow up can be demonstrated easily via methods of $[20,22]$. Some interesting dynamics which have been seen a lot in nature, such as stable steady states, chaotic dynamics and fast fluctuations can also be obtained.

Now let us conduct the linear stability analysis for the biologically feasible equilibriums which are the nonnegative steady states $\mathbf{u}^{(k)}, k=1,2,7,9$. We first linearize the system (1) along a nonnegative equilibrium solution $\overline{\mathbf{u}}=\left(\bar{u}_{1}, \bar{u}_{2}, \bar{u}_{3}\right)$ and the linear stability is determined by the eigenvalues of the Jacobian matrix

$$
\left.\mathbf{J}\right|_{\overline{\mathbf{u}}}=\left.\left(\begin{array}{ccc}
g_{1}+u_{1} \frac{\partial g_{1}}{\partial u_{1}} & u_{1} \frac{\partial g_{1}}{\partial u_{2}} & 0 \\
u_{2} \frac{\partial g_{2}}{\partial u_{1}} & g_{2}+u_{2} \frac{\partial g_{2}}{\partial u_{2}} & u_{2} \frac{\partial g_{2}}{\partial u_{3}} \\
0 & u_{3}^{2} \frac{\partial g_{3}}{\partial u_{2}} & 2 u_{3} g_{3}
\end{array}\right)\right|_{\overline{\mathbf{u}}} .
$$

For the nonnegative equilibriums $\mathbf{u}^{(k)}, k=1,2,7, \bar{u}_{3}=0$ and one of the eigenvalues of the Jacobian matrix is zero. Therefore these equilibria are not linearly stable in the three dimensional space. However, if we look at some subsystem, the answer might be different. For example, when we consider the subsystem of prey $u_{1}$ and middle predator $u_{2}$ while we assume $u_{3}=0$, the equilibrium $\mathbf{u}^{(\mathbf{7})}$ is linear stable for some parameters.

Thanks to the effect of the intra-species competition $b_{2}>0$, we have a nice stability result and a simple proof for the positive coexistence equilibrium solution $\mathbf{u}^{(9)}$. This result does not hold in the paper [21].

Theorem 2.1. Suppose that $\mathbf{u}^{(9)}$ is a positive equilibrium solution of the ODE system (1). If the parameters of the ODE system (1) satisfy

$$
w_{0} u_{2}^{+}<b_{1}\left(u_{1}^{+}+D_{0}\right)^{2} \text { and } w_{2} u_{3}^{+}<b_{2}\left(u_{2}^{+}+D_{2}\right)^{2}
$$

then the positive equilibrium solution $\mathbf{u}^{(9)}$ is locally asymptotically stable.

Proof. It is sufficient to show that the characteristic value of the Jacobian matrix (6) is 


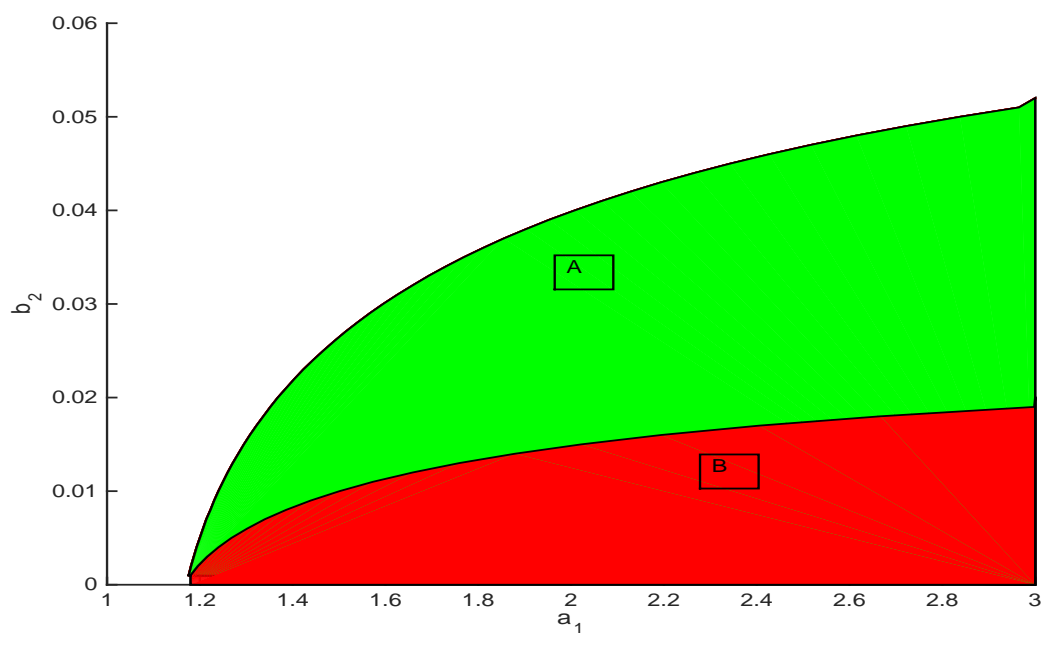

Figure 1: The linear stability space calculated in terms of the reproduction rate $a_{1}$ and intra-species competition $b_{2}$ among middle predators. All other parameters are given in (21) and (22) in section 5. The non-negative equilibrium $\mathbf{u}^{(9)}$ does not exist in the white blank region. But it exists in the colored regions (green A and red B). The top green region $\mathrm{A}$ is the linear stable region which satisfies the sufficient stability condition (7).

negative when $\overline{\mathbf{u}}=\mathbf{u}^{(9)}$. Since $g_{i}\left(\mathbf{u}^{(9)}\right)=0, i=1,2,3$, a direct calculation yields

$$
\left.J\right|_{\mathbf{u}^{(9)}}=\left(J_{i j}\right)=\left(\begin{array}{ccc}
u_{1}^{+}\left(-b_{1}+\frac{w_{0} u_{2}^{+}}{\left(u_{1}^{+}+D_{0}\right)^{2}}\right) & -\frac{u_{1}^{+} w_{0}}{u_{1}^{+}+D_{0}} & 0 \\
u_{2}^{+}\left(\frac{w_{1} D_{1}}{\left(u_{1}^{+}+D_{1}\right)^{2}}\right) & u_{2}^{+}\left(-b_{2}+\frac{w_{2} u_{3}^{+}}{\left(u_{2}^{+}+D_{2}\right)^{2}}\right) & -\frac{u_{2}^{+} w_{2}}{u_{2}^{+}+D_{2}} \\
0 & \frac{\left(u_{3}^{+}\right)^{2} w_{3}}{\left(u_{2}^{+}+D_{3}\right)^{2}} & 0
\end{array}\right)
$$

The characteristic polynomial of $\left.J\right|_{\mathbf{u}^{(9)}}$ is

$$
p(\lambda)=\lambda^{3}+A_{1} \lambda^{2}+A_{2} \lambda+A_{3},
$$

where

$$
\begin{gathered}
A_{1}=-J_{11}-J_{22}, \\
A_{2}=J_{11} J_{22}-J_{23} J_{32}-J_{21} J_{12}, \\
A_{3}=J_{11} J_{23} J_{32} .
\end{gathered}
$$

By the assumption (7), we have

$$
J_{11}<0, J_{12}<0, J_{22}<0, J_{23}<0, J_{21}>0 \text {, and } J_{32}>0 .
$$


It is easy to check that $A_{1}, A_{2}, A_{3}$ are positive and $A_{1} A_{2}-A_{3}=-J_{11}^{2} J_{22}+J_{11} J_{21} J_{12}-$ $J_{11} J_{22}^{2}+J_{22} J_{23} J_{32}+J_{22} J_{21} J_{12}>0$. Thus, the positive coexistence equilibrium solution $\mathbf{u}^{(9)}$ is locally asymptotically stable by the Routh-Hurwitz criterion.

Remark 2.2. The conditions (7) in theorem (2.1) are fulfilled for a range of parameters $a_{1}$ and $b_{2}$ while keeping other parameters as constants, for example, $w_{0}=1 ; w_{1}=2$; $w_{2}=0.55 ; w_{3}=1 ; D_{0}=10 ; D_{1}=10 ; D_{2}=10 ; D_{3}=20 ; a_{2}=1 ; b_{1}=0.05 ;$ and $a_{3}=0.03$. Numerically, we compute the regions of non-negative coexistence $\left(\mathbf{u}^{(9)}>0\right)$ and linear stability where conditions (7) are satisfied. Figure 1 presents the stable region (green region A) for Theorem 2.1 and coexistence region (green region A and red region B).

Remark 2.3. The conditions (7) in theorem (2.1) are sufficient but not necessary. For example, $\mathbf{u}^{(9)}$ is a positive stable equilibrium solution of (1) but the conditions (7) are not satisfied when $w_{0}=0.55 ; w_{1}=2 ; w_{2}=0.5 ; w_{3}=1.2 ; D_{0}=10 ; D_{1}=13 ; D_{2}=10 ; D_{3}=$ $20 ; a_{1}=2 ; a_{2}=0.8 ; a_{3}=0.04 ; b_{1}=0.15 ;$ and $b_{2}=0$. In fact, if $b_{2}=0$, the second inequality in (7) does not hold for any parameters. So the theorem is not applicable to the case studied in the paper [21], where $b_{2}=0$ and $k_{i j}=0$ for $i \neq j$. Numerically, we compute the linear stable regions by Routh-Hurwitz criterion and we compare it with linear stable region by conditions (7). Figure 2 presents three regions: (1) Red region B represents where the non-negative equilibrium $\mathbf{u}^{(9)}$ is linearly unstable. (2) Blue region $\mathrm{C}$ and green region A represent where the non-negative equilibrium $\mathbf{u}^{(9)}$ is linearly stable and that it satisfies Routh-Hurwitz criterion. (3) Green region A represents where the non-negative equilibrium $\mathbf{u}^{(9)}$ is linearly stable and that it satisfies both the Routh-Hurwitz criterion and the stability conditions (7).

\section{$3 \quad$ No Turing instability without cross-diffusion}

For the sake of simplicity, we denote

$$
\mathbf{K}(\mathbf{u})=\left(\begin{array}{c}
\left(k_{11}+k_{12} u_{2}\right) u_{1} \\
\left(k_{21} u_{1}+k_{22}+k_{23} u_{3}\right) u_{2} \\
\left(k_{32} u_{2}+k_{33}\right) u_{3}
\end{array}\right), \quad \mathbf{G}(\mathbf{u})=\left(\begin{array}{c}
u_{1} g_{1} \\
u_{2} g_{2} \\
u_{3}^{2} g_{3}
\end{array}\right),
$$

where $g_{1}, g_{2}, g_{3}$ are defined in the system of algebraic equations (3). Then the reactiondiffusion system (2) can be rewritten in matrix notation as:

$$
\left\{\begin{array}{l}
\frac{\partial \mathbf{u}}{\partial t}=\Delta \mathbf{K}(\mathbf{u})+\mathbf{G}(\mathbf{u}) \text { in } \Omega \times(0, \infty), \\
\frac{\partial \mathbf{u}}{\partial n}=0 \text { on } \partial \Omega \times(0, \infty), \\
\mathbf{u}(x, 0)=\left(u_{10}(x), u_{20}(x), u_{30}(x)\right)^{T} \text { in } \Omega .
\end{array}\right.
$$




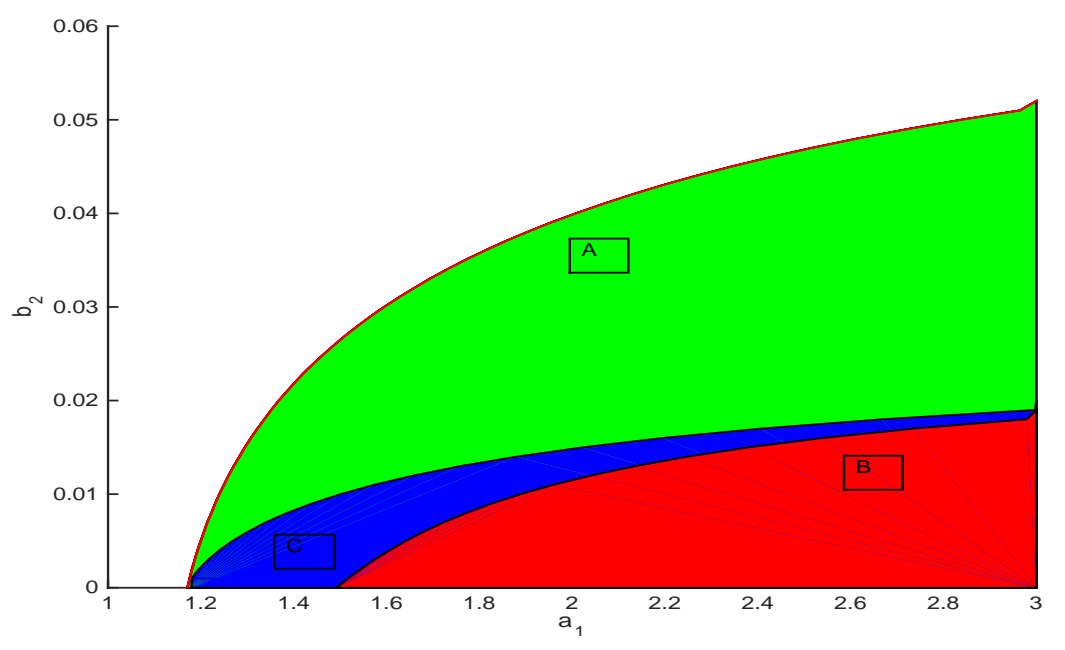

Figure 2: The linear stability space calculated in terms of the reproduction rate $a_{1}$ and intraspecies competition $b_{2}$ among middle predators. All other parameters are given in (21) and (22) in section 5. The green region $\mathrm{A}$ is the linear stable region which satisfies the sufficient stability conditions (7). Both the green region $\mathrm{A}$ and the blue region $\mathrm{C}$ are the linear stable regions which satisfy the Routh-Hurwitz criterion. The non-negative equilibrium $\mathbf{u}^{(9)}$ is linear unstable in the red region $\mathrm{B}$.

Linearizing the reaction-diffusion system (10) about a nonnegative equilibrium $\overline{\mathbf{u}}=\left(\bar{u}_{1}, \bar{u}_{2}, \bar{u}_{3}\right)$, we have

$$
\frac{\partial \boldsymbol{\Psi}}{\partial t}=\mathbf{K}_{\mathbf{u}}(\overline{\mathbf{u}}) \triangle \boldsymbol{\Psi}+\mathbf{G}_{\mathbf{u}}(\overline{\mathbf{u}}) \mathbf{\Psi}
$$

where $\boldsymbol{\Psi}=\left(\Psi_{1}, \Psi_{2}, \Psi_{3}\right)^{T}$ and

$$
\mathbf{K}_{\mathbf{u}}(\overline{\mathbf{u}})=\left(\begin{array}{ccc}
k_{11}+k_{12} \bar{u}_{2} & k_{12} \bar{u}_{1} & 0 \\
k_{21} \bar{u}_{2} & k_{21} \bar{u}_{1}+k_{22}+k_{23} \bar{u}_{3} & k_{23} \bar{u}_{2} \\
0 & k_{32} \bar{u}_{3} & k_{33}+k_{32} \bar{u}_{2}
\end{array}\right), \quad \mathbf{G}_{\mathbf{u}}(\overline{\mathbf{u}})=\left.\mathbf{J}\right|_{\overline{\mathbf{u}}} \text { in }(6) .
$$

Let $0=\mu_{1}<\mu_{2}<\mu_{3}<\cdots$ be the eigenvalues of the operator $-\triangle$ on $\Omega$ with the homogeneous Neumann boundary condition, and $E\left(\mu_{i}\right)$ be the eigenspace corresponding to $\mu_{i}$ in $C^{2}(\Omega)$. Let $\mathbf{X}=\left\{\mathbf{u} \in\left[C^{1}(\bar{\Omega})\right]^{3} \mid \partial_{n} \mathbf{u}=0\right.$ on $\left.\partial \Omega\right\},\left\{\phi_{i j}\right\}_{j=1,2, \cdots, \operatorname{dim} E\left(\mu_{i}\right)}$ be an orthonormal basis of $E\left(\mu_{i}\right)$, and $\mathbf{X}_{i j}=\left\{\mathbf{c} \phi_{i j} \mid \mathbf{c} \in \mathbf{R}^{3}\right\}$. Then

$$
\mathbf{X}=\bigoplus_{i=1}^{\infty} \mathbf{x}_{i} \quad \text { and } \quad \mathbf{x}_{i}=\bigoplus_{j=1}^{\operatorname{dim} E\left(\mu_{i}\right)} \mathbf{x}_{i j}
$$

For each $i \geq 1, \mathbf{X}_{i}$ is invariant under the operator $\mathbf{K}_{\mathbf{u}}(\overline{\mathbf{u}}) \triangle+\mathbf{G}_{\mathbf{u}}(\overline{\mathbf{u}})$. Then $\operatorname{PDE}$ (11) has a non trivial solution of the form $\boldsymbol{\Psi}=\mathbf{c} \phi \exp (\lambda t)$ if and only if $(\lambda, \mathbf{c})$ is an eigenpair for 
the matrix $-\mu_{i} \mathbf{K}_{\mathbf{u}}(\overline{\mathbf{u}})+\mathbf{G}_{\mathbf{u}}(\overline{\mathbf{u}})$, where $\mathbf{c}$ is a constant vector. Then the equilibrium $\overline{\mathbf{u}}$ is unstable if at least one eigenvalue $\lambda$ has a positive real part for some $\mu_{i}$.

For $\overline{\mathbf{u}}=\mathbf{u}^{(9)}, \mathbf{G}_{\mathbf{u}}(\overline{\mathbf{u}})=\left.\mathbf{J}\right|_{\mathbf{u}^{(9)}}$ in (8) and the characteristic polynomial of $-\mu_{i} \mathbf{K}_{\mathbf{u}}(\overline{\mathbf{u}})+$ $\mathbf{G}_{\mathbf{u}}(\overline{\mathbf{u}})$ for each $\mu_{i}$ is given by

$$
\rho_{i}(\lambda)=\lambda^{3}+B_{2 i} \lambda^{2}+B_{1 i} \lambda+B_{0 i}
$$

where

$$
-J_{22} k_{32} u_{2}^{+}-J_{11} k_{21} u_{1}^{+}+J_{23} k_{32} u_{3}^{+}-k_{11} J_{22}-J_{22} k_{33}+J_{21} k_{12} u_{1}^{+}-J_{11} k_{23} u_{3}^{+}-J_{11} k_{33}
$$

$\left.-J_{11} k_{22}\right) \mu_{i}+\left(k_{11} k_{23} u_{3}^{+}+k_{21} u_{1}^{+} k_{33}+k_{21} u_{1}^{+} k_{32} u_{2}^{+}+k_{12} u_{2}^{+} k_{23} u_{3}^{+}+k_{12} u_{2}^{+2} k_{32}+k_{12} u_{2}^{+} k_{22}\right.$

$\left.+k_{11} k_{21} u_{1}^{+}+k_{22} k_{33}+k_{12} u_{2}^{+} k_{33}+k_{11} k_{32} u_{2}^{+}+k_{11} k_{22}+k_{22} k_{32} u_{2}^{+}+k_{23} u_{3}^{+} k_{33}+k_{11} k_{33}\right) \mu_{i}^{2}$

$$
\begin{gathered}
\left.J_{11} J_{22} k_{32} u_{2}^{+}-J_{21} J_{12} k_{33}-J_{11} J_{23} k_{32} u_{3}^{+}-J_{11} k_{23} u_{2}^{+} J_{32}-k_{12} u_{2}^{+} J_{23} J_{32}\right) \mu_{i} \\
+\left(-J_{11} k_{22} k_{33}+J_{21} k_{12} u_{1}^{+} k_{33}-J_{11} k_{22} k_{32} u_{2}^{+}+k_{11} J_{23} k_{32} u_{3}^{+}+k_{21} u_{2}^{+2} J_{12} k_{32}-k_{11} J_{22} k_{33}\right. \\
-k_{12} u_{2}^{+2} J_{22} k_{32}+k_{12} u_{2}^{+2} k_{23} J_{32}-J_{11} k_{21} u_{1}^{+} k_{33}+J_{21} k_{12} u_{1}^{+} k_{32} u_{2}^{+}-J_{11} k_{23} u_{3}^{+} k_{33} \\
-k_{11} J_{22} k_{32} u_{2}^{+}+k_{11} k_{23} u_{2}^{+} J_{32}+k_{21} u_{2}^{+} J_{12} k_{33}+k_{12} u_{2}^{+} J_{23} k_{32} u_{3}^{+}-k_{12} u_{2}^{+} J_{22} k_{33} \\
\left.-J_{11} k_{21} u_{1}^{+} k_{32} u_{2}^{+}\right) \mu_{i}{ }^{2}+\left(k_{11} k_{22} k_{33}+k_{11} k_{23} u_{3}^{+} k_{33}+k_{12} u_{2}^{+} k_{22} k_{33}+k_{11} k_{21} u_{1}^{+} k_{32} u_{2}^{+}\right. \\
\left.+k_{12} u_{2}^{+} k_{23} u_{3}^{+} k_{33}+k_{12} u_{2}^{+2} k_{22} k_{32}+k_{11} k_{21} u_{1}^{+} k_{33}+k_{11} k_{22} k_{32} u_{2}^{+}\right) \mu_{i}{ }^{3}
\end{gathered}
$$$$
B_{0 i}=J_{11} J_{23} J_{32}+\left(J_{11} J_{22} k_{33}-J_{21} J_{12} k_{32} u_{2}^{+}-k_{11} J_{23} J_{32}+\right.
$$

Let $\lambda_{1 i}, \lambda_{2 i}, \lambda_{3 i}$ be the three roots of (12)

$$
\rho_{i}(\lambda)=\lambda^{3}+B_{2 i} \lambda^{2}+B_{1 i} \lambda+B_{0 i}=0 .
$$

In order to obtain the stability of $\overline{\mathbf{u}}$, we need to show that there exists a positive constant $\delta$ such that

$$
\operatorname{Re}\left\{\lambda_{1 i}\right\}, \operatorname{Re}\left\{\lambda_{2 i}\right\}, \operatorname{Re}\left\{\lambda_{3 i}\right\}<-\delta \text { for all } i \geq 1 .
$$

Next we use the Routh-Hurwitz criterion to study stability [9]. For a third-order polynomial to be linearly stable, i.e. to have all its roots with negative real parts, it is necessary and sufficient that

$$
B_{j i}>0, \text { and } B_{1 i} B_{2 i}>B_{0 i}, \text { for } j=1,2,3 \text { and all } i .
$$

The aim of the following theorem is to prove that the diffusion alone (without cross-diffusion, i.e. $k_{21}=k_{12}=k_{32}=k_{23}=0$ ) can not drive instability for this food chain model, i.e., Turing instability does not occur in the three species food chain model without crossdiffusion. 
Theorem 3.1. Suppose that (7) holds and $k_{21}=k_{12}=k_{32}=k_{23}=0$. Then the positive coexistence equilibrium solution $\overline{\mathbf{u}}=\mathbf{u}^{(9)}$ of (10) is locally asymptotically stable.

Proof. Substituting $k_{21}=k_{12}=k_{32}=k_{23}=0$ into $B_{2 i}, B_{1 i}$ and $B_{0 i}$, we have

$$
\begin{gathered}
B_{2 i}=-J_{22}-J_{11}+\left(k_{11}+k_{33}+k_{22}\right) \mu_{i} \\
B_{1 i}=-J_{21} J_{12}+J_{11} J_{22}-J_{23} J_{32}+\left(-J_{11} k_{33}-J_{22} k_{33}-k_{11} J_{22}-J_{11} k_{22}\right) \mu_{i} \\
+\left(k_{11} k_{33}+k_{22} k_{33}+k_{11} k_{22}\right) \mu_{i}{ }^{2} \\
B_{0 i}=J_{11} J_{23} J_{32}+\left(-J_{21} J_{12} k_{33}-k_{11} J_{23} J_{32}+J_{11} J_{22} k_{33}\right) \mu_{i} \\
+\left(-k_{11} J_{22} k_{33}-J_{11} k_{22} k_{33}\right) \mu_{i}{ }^{2}+k_{11} k_{22} k_{33} \mu_{i}{ }^{3}
\end{gathered}
$$

By simply applying the sign of $J_{i j}$ in $(9)$ and $k_{i j}>0$, we have $B_{2 i}>0, B_{1 i}>0, B_{0 i}>0$. A direct calculation shows that

$$
\begin{gathered}
B_{2 i} B_{1 i}-B_{0 i}=\left(-J_{22}-J_{11}\right)\left(-J_{21} J_{12}+J_{11} J_{22}-J_{23} J_{32}\right)-J_{11} J_{23} J_{32} \\
+\left(\left(-J_{22}-J_{11}\right)\left(-J_{11} k_{33}-J_{22} k_{33}-k_{11} J_{22}-J_{11} k_{22}\right)+\left(k_{11}+k_{33}+k_{22}\right)\left(-J_{21} J_{12}\right.\right. \\
\left.\left.+J_{11} J_{22}-J_{23} J_{32}\right)+J_{21} J_{12} k_{33}+k_{11} J_{23} J_{32}-J_{11} J_{22} k_{33}\right) \mu_{i}+\left(( - J _ { 2 2 } - J _ { 1 1 } ) \left(k_{11} k_{33}\right.\right. \\
\left.+k_{22} k_{33}+k_{11} k_{22}\right)+\left(k_{11}+k_{33}+k_{22}\right)\left(-J_{11} k_{33}-J_{22} k_{33}-k_{11} J_{22}-J_{11} k_{22}\right) \\
\left.+k_{11} J_{22} k_{33}+J_{11} k_{22} k_{33}\right) \mu_{i}^{2} \\
+\left(\left(k_{11}+k_{33}+k_{22}\right)\left(k_{11} k_{33}+k_{22} k_{33}+k_{11} k_{22}\right)-k_{11} k_{22} k_{33}\right) \mu_{i}^{3}>0
\end{gathered}
$$

for all $i \geq 1$. It follows from the Routh-Hurwitz criterion (17) that, all the three roots $\lambda_{1 i}$, $\lambda_{2 i}, \lambda_{3 i}$ of $\rho_{i}(\lambda)=0$ have negative real parts for each $i \geq 1$.

Let $\lambda=\mu_{i} \xi$, then

$$
\rho_{i}(\lambda)=\mu_{i}^{3} \xi^{3}+B_{2 i} \mu_{i}^{2} \xi^{2}+B_{1 i} \mu_{i} \xi+B_{0 i} \equiv \tilde{\rho}_{i}(\xi) .
$$

Since $\mu_{i} \rightarrow \infty$ as $i \rightarrow \infty$, we have

$$
\bar{\rho}(\xi)=\lim _{i \rightarrow \infty} \frac{\tilde{\rho}_{i}(\xi)}{\mu_{i}^{3}}=\xi^{3}+\left(k_{33}+k_{22}+k_{11}\right) \xi^{2}+\left(k_{22} k_{33}+k_{11} k_{33}+k_{11} k_{22}\right) \xi+k_{11} k_{22} k_{33} .
$$

Applying the Routh-Hurwitz criterion it follows that the three roots $\xi_{1}, \xi_{2}, \xi_{3}$ of $\bar{\rho}(\xi)=0$ all have negative real parts. Thus, there exists a positive constant $\bar{\delta}$ such that $\operatorname{Re}\left\{\xi_{1}\right\}$, $\operatorname{Re}\left\{\xi_{2}\right\}, \operatorname{Re}\left\{\xi_{3}\right\} \leq-2 \bar{\delta}$. By continuity, we see that there exists $i_{0} \geq 1$ such that $\mu_{i_{0}}>1$ and the three roots $\xi_{i 1}, \xi_{i 2}, \xi_{i 3}$ of $\tilde{\rho}_{i}(\xi)=0$ satisfy $\operatorname{Re}\left\{\xi_{i 1}\right\}, \operatorname{Re}\left\{\xi_{i 2}\right\}, \operatorname{Re}\left\{\xi_{i 3}\right\} \leq-\bar{\delta}$ for any $i \gtrsim i_{0}$. Then $\operatorname{Re}\left\{\lambda_{i 1}\right\}, \operatorname{Re}\left\{\lambda_{i 2}\right\}, \operatorname{Re}\left\{\lambda_{i 3}\right\} \leq-\mu_{i} \bar{\delta} \leq-\mu_{i 0} \bar{\delta} \leq-\bar{\delta}$ for any $i \geq i_{0}$. Let $-\tilde{\delta}=\max _{1 \leq i \leq i_{0}}\left\{\operatorname{Re}\left\{\lambda_{i 1}\right\}, \operatorname{Re}\left\{\lambda_{i 2}\right\}, \operatorname{Re}\left\{\lambda_{i 3}\right\}\right\}$ and $\delta=\min \{\tilde{\delta}, \bar{\delta}\}$. Then

$$
\operatorname{Re}\left\{\lambda_{1 i}\right\}, \operatorname{Re}\left\{\lambda_{2 i}\right\}, \operatorname{Re}\left\{\lambda_{3 i}\right\}<-\delta \text { for all } i \geq 1 .
$$

Consequently the positive equilibrium $\mathbf{u}^{(9)}$ is locally asymptotically stable. 


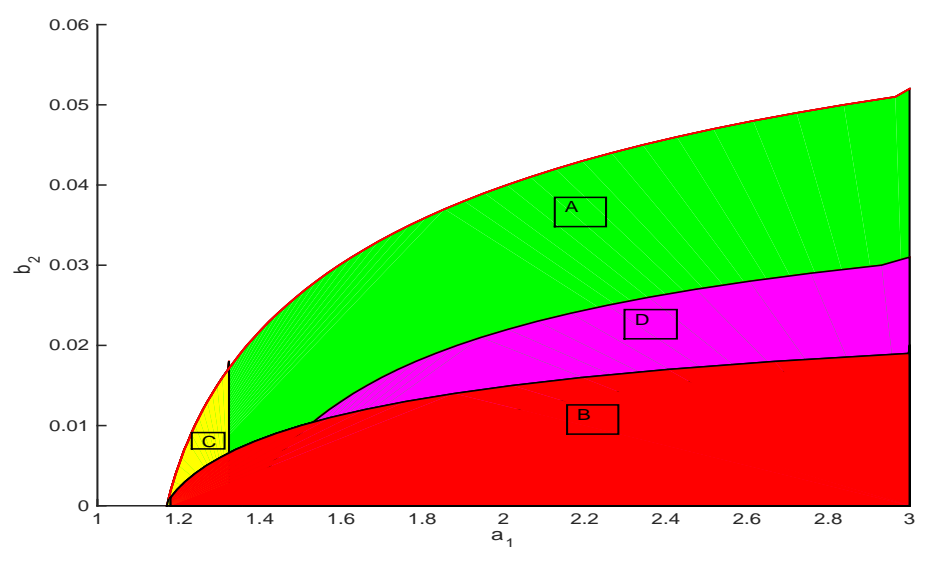

Figure 3: The Turing instability conditions are calculated in terms of the reproduction rate $a_{1}$ and intra-species competition $b_{2}$ among middle predators. All other parameters are given in (21) and (22) in section 5. The non-negative equilibrium $\mathbf{u}^{(9)}$ exists in all four colored regions ( $\mathrm{A}, \mathrm{B}, \mathrm{C}$, and $\mathrm{D})$. The non-negative equilibrium $\mathbf{u}^{(9)}$ is linear stable in the top three regions (green A, yellow C, and magenta D) where conditions (7) are satisfied. Turing instability conditions (7) and (18) are satisfied in the yellow region C. Turing instability conditions (7) and (19) are satisfied only in the magenta region D.

Note that $B_{2 i}>0$ in (13), $B_{1 i}>0$ in (14), $B_{0 i}>0$ in (15), and $B_{2 i} B_{1 i}-B_{0 i}>0$ if $k_{21}=k_{32}=0$ since the possible negative terms all involve either $k_{21}$ or $k_{32}$. By the same arguments as in Theorem 3.1, we have

Theorem 3.2. Suppose that (7) holds and $k_{21}=k_{32}=0$. Then the positive equilibrium $\overline{\mathbf{u}}=\mathbf{u}^{(9)}$ of (10) is locally asymptotically stable.

\section{Turing Instability driven by cross-diffusion}

The Turing instability [14] refers to "diffusion-driven instability, "i.e., the stability of the positive equilibrium $\overline{\mathbf{u}}=\mathbf{u}^{(9)}$ changing from stable, for the ODE dynamics (1), to unstable, for the PDE dynamics (10). From Theorem 3.1 we see that only adding self-crossing diffusion to the ODE system (1), the positive equilibrium is also locally stable, which means that Turing instability has not happened. From Theorem 3.2, crossing diffusion between middle specialist predator and prey $\left(k_{12}>0, k_{23}>0\right)$ can not drive instability either. Here we are going to give sufficient conditions on cross-diffusion which drives the instability. $k_{21}$ and $k_{32}$ can be chosen as variation parameters, whereas the other constants are fixed.

Theorem 4.1. (1) Suppose that the conditions (7) and the following inequality are sat- 
isfied

$$
b_{1}\left(u_{1}^{+}+D_{0}\right)^{2}<w_{0} u_{2}^{+} \frac{\left(2 u_{1}^{+}+D_{0}\right)}{u_{1}^{+}} .
$$

Let $k_{21}$ be the variation parameter. Then there exists a positive constant $\delta_{21}$ such that when $k_{21}>\delta_{21}$, the equilibrium $\mathbf{u}(x, t)=\mathbf{u}^{(9)}$ is linearly unstable for some domain $\Omega$.

(2) Suppose that the conditions (7) and the following inequality are satisfied

$$
\left(J_{11} J_{22}-J_{21} J_{12}\right) u_{2}^{+}<J_{11} J_{23} u_{3}^{+}
$$

Let $k_{32}$ be the variation parameter. Then there exists a positive constant $\delta_{32}$ such that when $k_{32}>\delta_{32}$, the equilibrium $\mathbf{u}(x, t)=\mathbf{u}^{(9)}$ is linearly unstable for some domain $\Omega$.

Remark 4.2. (a) $k_{21}$ and $k_{32}$ can be chosen as variation parameters because the number of sign changes for the polynomial (20) could be bigger than one for large values of $k_{21}$ or $k_{32}$. By Descartes' Rule, the polynomial (20) could have positive roots which lead to linear instability.

(b) The conditions (7) are compatible with the condition (18). The conditions (7) are also compatible with the condition (19). We refer those conditions satisfying either (7) and (18) or (7) and (19) as the Turing instability conditions. We will use examples to illustrate the existence of parameters which satisfy the Turing instability conditions.

(c) In fact, if the parameters are given as in (21) and (22) in section 5 with the assumption of the condition (7), the region of $a_{1}$ and $b_{2}$ satisfying the Turing instability conditions (7) and (18) is shown as the yellow region $\mathrm{C}$ in Figure 3 and the region of the Turing instability conditions (7) and (19) is shown as the magenta region D.

Proof. We will use an argument similar to those in paper [24] to prove the existence of Turing instability. By the assumptions (7) and the sign of $J_{i j}$ in (9), we have $B_{2 i}>0$ and $B_{1 i}>0$. By the Routh-Hurwitz criteria (17), the stable equilibrium $\mathbf{u}^{(\mathbf{9})}$ will be destabilized by diffusion if $B_{0 i}<0$ for some $i$.

Denote $A(\mu)=-\mu \mathbf{K}_{\mathbf{u}}(\overline{\mathbf{u}})+\mathbf{G}_{\mathbf{u}}(\overline{\mathbf{u}})$. We have $B_{0 i}=-\operatorname{det}\left(A\left(\mu_{i}\right)\right)$. By the direct computations we have

$$
\operatorname{det}(A(\mu))=-\left(C_{3} \mu^{3}+C_{2} \mu^{2}+C_{1} \mu+C_{0}\right),
$$

where

$$
\begin{gathered}
C_{0}=J_{11} J_{23} J_{32} ; \\
C_{1}=\left(J_{11} J_{22} u_{2}^{+}-J_{21} J_{12} u_{2}^{+}-J_{11} J_{23} u_{3}^{+}\right) k_{32}+
\end{gathered}
$$




$$
\begin{gathered}
J_{11} J_{22} k_{33}-k_{11} J_{23} J_{32}-J_{21} J_{12} k_{33}-J_{11} k_{23} u_{2}^{+} J_{32}-k_{12} u_{2}^{+} J_{23} J_{32} ; \\
C_{2}=\left(\left(J_{12} u_{2}^{+}-J_{11} u_{1}^{+}\right) k_{32} u_{2}^{+}+\left(J_{12} u_{2}^{+}-J_{11} u_{1}^{+}\right) k_{33}\right) k_{21} \\
-J_{11} k_{22} k_{32} u_{2}^{+}+k_{11} J_{23} k_{32} u_{3}^{+}-k_{12} u_{2}^{+2} J_{22} k_{32}+J_{21} k_{12} u_{1}^{+} k_{32} u_{2}^{+}-k_{11} J_{22} k_{32} u_{2}^{+} \\
-J_{11} k_{22} k_{33}+J_{21} k_{12} u_{1}^{+} k_{33}-k_{11} J_{22} k_{33}+k_{12} u_{2}^{+2} k_{23} J_{32}-J_{11} k_{23} u_{3}^{+} k_{33} \\
+k_{11} k_{23} u_{2}^{+} J_{32}+k_{12} u_{2}^{+} J_{23} k_{32} u_{3}^{+}-k_{12} u_{2}^{+} J_{22} k_{33} ; \\
C_{3}=k_{11} k_{22} k_{33}+k_{11} k_{23} u_{3}^{+} k_{33}+k_{12} u_{2}^{+} k_{22} k_{33}+k_{11} k_{21} u_{1}^{+} k_{32} u_{2}^{+}+k_{12} u_{2}^{+} k_{23} u_{3}^{+} k_{33} \\
+k_{12} u_{2}^{+2} k_{22} k_{32}+k_{11} k_{21} u_{1}^{+} k_{33}+k_{11} k_{22} k_{32} u_{2}^{+} .
\end{gathered}
$$

By using the assumptions (7) and the sign of $J_{i j}$ in (9), it is easy to show that $C_{0}>0$ and $C_{3}>0$.

\section{CASE 1: $k_{21}$ is the variation parameter.}

Because each root of the algebraic equation (20) is a continuous function of the variation parameter $k_{21}$, it is easy to prove that equation (20) has three real roots $\mu_{1}^{(i)}=\mu_{1}^{(i)}\left(k_{21}\right)$, $i=1,2,3$ when $k_{21}$ goes to infinity and they are $\lim _{k_{21} \rightarrow \infty} \mu_{1}^{(1)}\left(k_{21}\right)=\lim _{k_{21} \rightarrow \infty} \mu_{1}^{(2)}\left(k_{21}\right)=$ 0 and

$$
\lim _{k_{21} \rightarrow \infty} \mu_{1}^{(3)}\left(k_{21}\right)=\frac{J_{11} u_{1}^{+}-J_{12} u_{2}^{+}}{k_{11} u_{1}^{+}} .
$$

The inequality (18) implies that $J_{11} u_{1}^{+}-J_{12} u_{2}^{+}>0$. Therefore, the root $\lim _{k_{21} \rightarrow \infty} \mu_{1}^{(3)}\left(k_{21}\right)$ is positive. By continuity, there exists a positive constant $\delta_{21}$ such that when $k_{21}>\delta_{21}$, $C_{2}<0$ and $\operatorname{det}(A(\mu))=0$ has three real roots. Because $C_{3}>0$ and $C_{0}>0$, the number of sign changes of (20) is exactly two. Therefore by Descartes' rule, the three real roots have the following properties:

(i) $-\infty<\mu_{1}^{(1)}<0<\mu_{1}^{(2)}<\mu_{1}^{(3)}<\infty$;

(ii) $\operatorname{det}(A(\mu))>0$ if $\mu \in\left(-\infty, \mu_{1}^{(1)}\right) \bigcup\left(\mu_{1}^{(2)}, \mu_{1}^{(3)}\right)$;

(iii) $\operatorname{det}(A(\mu))<0$ if $\mu \in\left(\mu_{1}^{(1)}, \mu_{1}^{(2)}\right) \bigcup\left(\mu_{1}^{(3)}, \infty\right)$.

If $\mu_{i} \in\left(\mu_{1}^{(2)}, \mu_{1}^{(3)}\right)$ for some $i$, then $\operatorname{det}\left(A\left(\mu_{i}\right)\right)>0$ by (ii), and consequently $B_{0 i}=$ $-\operatorname{det}\left(A\left(\mu_{i}\right)\right)<0$. The number of sign changes of the characteristic polynomial (12) $\rho(\lambda)=\lambda^{3}+B_{2 i} \lambda^{2}+B_{1 i} \lambda+B_{0 i}$ is either one or three. By Descartes' rule, the characteristic polynomial (12) has at least one positive root. Hence, the equilibrium $\mathbf{u}^{(9)}$ of (10) is linearly unstable for any domain $\Omega$ on which at least one eigenvalue $\mu_{i}$ of $-\triangle$ is in the 
interval $\left(\mu_{1}^{(2)}, \mu_{1}^{(3)}\right)$.

\section{CASE 2: $k_{32}$ is the variation parameter.}

Note that the inequality (19) implies that $C_{1}<0$ for large $k_{32}$. Because each root of the algebraic equation (20) is a continuous function of the variation parameter $k_{32}$, it is easy to prove that equation (20) has three real roots $\mu_{2}^{(i)}=\mu_{2}^{(i)}\left(k_{32}\right), i=1,2,3$ when $k_{32}$ goes to infinity and they are $\lim _{k_{32} \rightarrow \infty} \mu_{2}^{(1)}\left(k_{32}\right)<0$ and $\lim _{k_{32} \rightarrow \infty} \mu_{2}^{(2)}\left(k_{32}\right)=0$ and $\lim _{k_{32} \rightarrow \infty} \mu_{2}^{(3)}\left(k_{32}\right)>0$. This is done by applying the Descartes' Rule to the limit case when $k_{32} \rightarrow \infty$.

By continuity, there exists a positive constant $\delta_{32}$ such that when $k_{32}>\delta_{32}, C_{1}<0$ and $\operatorname{det}(A(\mu))=0$ has three real roots. Because $C_{3}>0$ and $C_{0}>0$, the number of sign changes of (20) is exactly two. Therefore by the Descartes' rule, the three real roots have the following properties:

(i) $-\infty<\mu_{2}^{(1)}<0<\mu_{2}^{(2)}<\mu_{2}^{(3)}<\infty$;

(ii) $\operatorname{det}(A(\mu))>0$ if $\mu \in\left(-\infty, \mu_{2}^{(1)}\right) \bigcup\left(\mu_{2}^{(2)}, \mu_{2}^{(3)}\right)$;

(iii) $\operatorname{det}(A(\mu))<0$ if $\mu \in\left(\mu_{2}^{(1)}, \mu_{2}^{(2)}\right) \bigcup\left(\mu_{2}^{(3)}, \infty\right)$.

If $\mu_{i} \in\left(\mu_{2}^{(2)}, \mu_{2}^{(3)}\right)$ for some $i$, then $\operatorname{det}\left(A\left(\mu_{i}\right)\right)>0$, and consequently $B_{0 i}=-\operatorname{det}\left(A\left(\mu_{i}\right)\right)<0$ and $\rho_{i}(0)=B_{0 i}<0$. By similar argument as in case 1 , the characteristic polynomial (12) has at least one positive root. Hence, the equilibrium $\mathbf{u}^{(9)}$ of (10) is linearly unstable for any domain $\Omega$ in which at least one eigenvalue $\mu_{i}$ of $-\triangle$ is in the interval $\left(\mu_{2}^{(2)}, \mu_{2}^{(3)}\right)$.

Remark 4.3. Biological interpretation: In our three-species food chain model, the third species (the generalist predator) preys on the second species (the specialist predator) and simultaneously the second one preys on the first one (the prey). Biologically, a cross diffusion coefficient $k_{12}$ in the system (2) means that the prey $u_{1}$ intends to keep away from its predators $u_{2}$. The cross diffusion $k_{21}$ represents the tendency of the predator $u_{2}$ to chase its prey. Positive cross diffusion coefficient implies that one species takes advantage of the strategies that they tends to move in the direction of lower concentration of another species. However, negative cross diffusion coefficient means that one species would take advantage of moving in the direction of higher concentration of another species. Similar biological meaning is applied to the cross diffusion coefficients $k_{23}$ and $k_{32}$. In this paper, we study only the case when the species move in the direction of lower concentration of another species, i.e. $k_{i j} \geq 0$.

Predator $u_{2}$ can be treated as a prey with respect to the predator $u_{3}$. When the positive equilibrium solution $\mathbf{u}^{(\mathbf{9})}$ satisfies the condition (7), the strategies $\left(k_{12}\right.$ or $\left.k_{23}\right)$ that 
the prey $u_{1}$ and the prey $u_{2}$ used are not able to affect the stability of the equilibrium (long term population distribution), i.e. $k_{12}$ or $k_{23}$ can not destablize the stability of the equilibrium. Keeping away from predators can not break the stability of the equilibrium. However, the changing of the strategies of predators chasing prey may affect the stability of the equilibrium. We discover that the positive steady state of the three species food chain can be broken by the reaction diffusion among two species on the chain.

When the positive equilibrium solution $\mathbf{u}^{(\mathbf{9})}$ satisfies the conditions (7) and (18), the cross diffusion $k_{21}$ can drive the stable equilibrium away. In this case, the first species (the prey) are assumed to reproduce exponentially unless subject to intra-species competition and predation. The exponential growth is modeled by the term $a_{1} u_{1}$ in system (2). The level of intra-species competition among the first species is proportional to its population size by the term $b_{1} u_{1}$. The functional response of the first species (the prey) to the specialist predator is the Holling type II functional response which takes the environment protection into consideration. At the positive equilibrium solutions, if the population of the second

species $u_{2}^{+}$is bounded by a function of $u_{1}^{+}$, i.e. $b_{1}\left(u_{1}^{+}+D_{0}\right)^{2} \frac{u_{1}^{+}}{\left(2 u_{1}^{+}+D_{0}\right)}<w_{0} u_{2}^{+}<b_{1}\left(u_{1}^{+}+\right.$ $\left.D_{0}\right)^{2}$, the large cross-diffusion of the second species due to the first species $\left(k_{21}\right)$ can break the stability. In other words, if the predator (second species) has a dominate effect on the decreasing of the prey (first species) such as the maximum per capita reduction rate $w_{0}$ is appropriate, then the predator (the second species) with large cross-diffusion can destabilize the stable equilibrium.

\section{$5 \quad$ Numerical Results}

In this section, we use numerical simulations to study the longterm behavior of the solutions to our three species food chain model.

\subsection{Diversity of Solutions for ODE system (1) without Competition among the Specialist Predators $\left(b_{2}=0\right)$}

The ODE system (1) was studied by Upadhyay, Iyengar and Rai [17] in the case $b_{2}=0$. It was shown that the system has a very diversity of dynamical behaviors. Chaotic solutions occur in various ranges of the parameter space. In addition to chaotic solutions, there are states of extinction for certain species, stable equilibrium solutions, and limit cycles. We want to illustrate how the longterm behavior of the solution can vary from one state to another state when the growth rate of prey changes. A bifurcation curve is on $a_{1}$ described in table 1.

In this subsection, the numerical simulation is carried out by using ODE45 function in MATLAB (R2014b). Since there are 13 parameters, the search for the diversity of solutions was found by fixing some common parameters and varying the growth rate $a_{1}$ of the prey. 
In addition to $b_{2}=0$, the other common parametric values for this subsection are

$$
\begin{gathered}
a_{2}=1 ; a_{3}=0.03 ; b_{1}=0.05 ; w_{0}=1 ; w_{1}=2 ; w_{2}=0.55 ; w_{3}=1 ; \\
D_{0}=10 ; D_{1}=10 ; D_{2}=10 ; D_{3}=20 .
\end{gathered}
$$

Then there are at most four nonnegative equilibriums which depends on $a_{1}$. They are

$$
\mathbf{u}^{(\mathbf{1})}=(0,0,0) ; \mathbf{u}^{(\mathbf{2})}=\left(20 a_{1}, 0,0\right) \text { for } a_{1}>0 ; \mathbf{u}^{(\mathbf{7})}=\left(10,20 a_{1}-10,0\right) \text { for } a_{1}>0.5 ;
$$

and when $a_{1} \geq \frac{7}{6}$

$\mathbf{u}^{(\mathbf{9})}=\left(10 a_{1}-5+10 \sqrt{a_{1}^{2}+a_{1}-\frac{29}{12}}, \frac{40}{3},-\frac{1400}{33}+\frac{2800\left(10 a_{1}-5+10 \sqrt{a_{1}^{2}+a_{1}-\frac{29}{12}}\right)}{33\left(10 a_{1}+5+10 \sqrt{a_{1}^{2}+a_{1}-\frac{29}{12}}\right)}\right)$.

In table 1, we list the detailed information about the nonnegative equilibriums and the corresponding parameter spaces. A typical solution is computed for a specific growth rate of the prey. All solutions start the same initial point $\mathbf{u}=(15,13,9)$. We use ODE45 with error tolerance less than $10^{-4}$ to solve the initial value problem of the ODE system (1). The time interval is chosen appropriately for a better display of the solution behaviors. We put them into the following cases and they are illustrated in table 1.

- $(0,0,0)$ is always a trivial equilibrium but no solution will converge to it when the growth rate $a_{1}$ of prey is greater than zero. Not all species will extinct.

- When $0<a_{1} \leq \frac{1}{2}$, solutions starting from any positive initial condition converge to the nonnegative equilibrium $\mathbf{u}^{(\mathbf{2})}$. For $a_{1}=0.45, \mathbf{u} \rightarrow \mathbf{u}^{(\mathbf{2})}=(9,0,0)$. Only prey will survive after a long time.

- When $\frac{1}{2}<a_{1} \leq \frac{7}{6}$, solutions of the ODE system converge to the nonnegative equilib$\operatorname{rium} \mathbf{u}^{(\mathbf{7})}$. For $a_{1}=1, \mathbf{u} \rightarrow \mathbf{u}^{(\mathbf{7})}=(10,10,0)$. Only the top generalist predator will be extinct.

- When $\frac{7}{6}<a_{1} \leq 1.492$, solutions of the ODE system converge to the nonnegative equilibrium $\mathbf{u}^{(\mathbf{9})}$, which is linearly stable. For $a_{1}=1.3, \mathbf{u} \rightarrow \mathbf{u}^{(\mathbf{9})}=(15.5719,13.3333,9.2439)$. Three species will coexist and the population is stable after a long time. But when $a_{1}$ is near 1.492, it may takes a long time to approach the equilibrium solution.

- When $1.492<a_{1}$, the solutions of the ODE system experience a fluctuation. Limit cycles and chaotic solutions can occur. Numerically, limit cycles clearly happen for $1.5 \leq a_{1}<1.6$. Chaotic solutions clearly occur for $1.6<a_{1}<2.1$. Then the phenomenon of the limit cycles happens again. Some blow-up solution can happen when $a_{1}$ is large. 


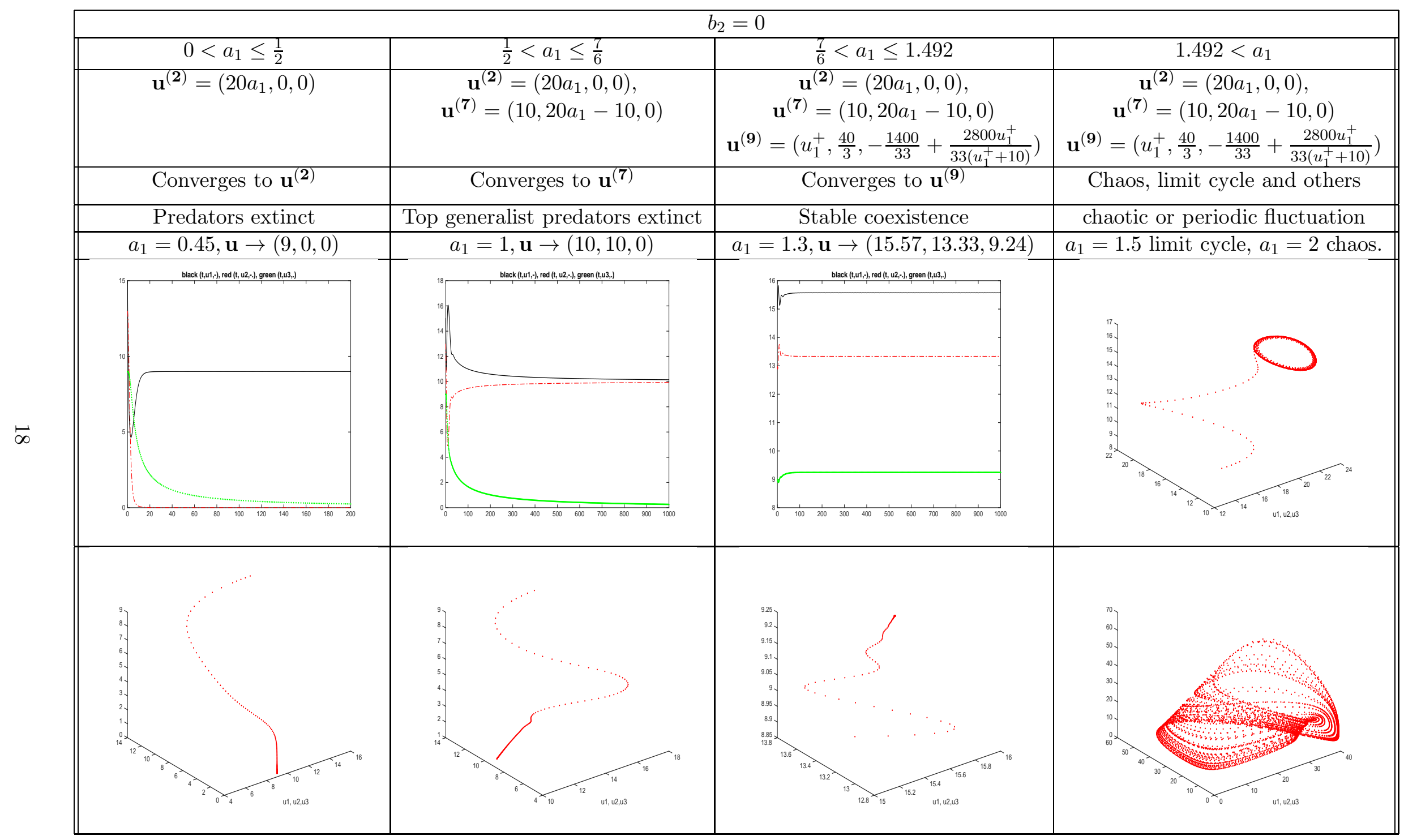

Table 1: The values of other parameters are given in $(21)$ and $(22) \cdot u_{1}^{+}=10 a_{1}-5+10 \sqrt{a_{1}^{2}+a_{1}-\frac{29}{12}}$ in $\mathbf{u}^{(\mathbf{9})}$. Solutions converge to an equilibrium, a limit cycle or they experience chaotic. Graphs are either solution curves over time or phase portrait. 


\subsection{Affect of the Intra-species Competition among the Specialist Preda- tors $\left(b_{2} \neq 0\right)$}

In this subsection, we are going to illustrate numerically how the intra-species competition among the specialist predators will change the longterm behavior of a solution. We consider three cases: (1) the stable coexistence for $a_{1}=1.3$; (2) the limit cycle for $a_{1}=1.5$; (3) the chaos for $a_{1}=2$. By the continuity of solutions on parameters, when $b_{2}$ is very small, the behavior of solution will similar to those solutions in table (1) for $b_{2}=0$. The numerical values of the parameters are taken from (21) and (22). To have a better view of simulation, the initial conditions are chosen appropriately (close to the ending point). The numerical graphs are illustrated in table 2 for the following cases.

- When $a_{1}=1.3$, the solutions converge to the stable equilibrium $\mathbf{u}^{(\mathbf{9})}$ for $0 \leq b_{2}<$ 0.016. The solutions converge to equilibrium $\mathbf{u}^{(\mathbf{7})}$ for $b_{2}>0.017$. In particular,when $b_{2}=0.01, \mathbf{u}^{(\mathbf{9})}=[15.5719,13.3333,3.5873] ;$ when $b_{2}=0.2, \mathbf{u}^{(\mathbf{7})}=[24.7782,2.1246,0]$; when $b_{2}=2, \mathbf{u}^{(\mathbf{7})}=[25.8767,0.2213,0]$. As the intra-species competition among specialist predator $b_{2}$ increases, the stability of equilibrium changes and the population density of specialist predator is decreasing.

- When $a_{1}=1.5$, the equilibrium $\mathbf{u}^{(\mathbf{9})}$ is unstable and limit cycle occurs for $b_{2}=0$. When $b_{2}$ increases from 0 to a small positive number, the unstable equilibrium $\mathbf{u}^{(\mathbf{9})}$ becomes to a stable equilibrium. For example, when $b_{2}=0.01$, the equilibrium $\mathbf{u}^{(\mathbf{9})}=[21.5470,13.3333,9.8718]$ is stable. While the intra-species competition among specialist predator $b_{2}$ keeps on increasing, the positive equilibrium $\mathbf{u}^{(\mathbf{9})}$ will disappear and the solutions will converge to $\mathbf{u}^{(\boldsymbol{7})}$. For example when $b_{2}=0.1$, the solutions will converge to $\mathbf{u}^{(7)}=[27.5110,4.6682,0]$.

- When $a_{1}=2$, the equilibrium $\mathbf{u}^{(\mathbf{9})}$ is unstable and chaos occurs for $b_{2}=0$. When $b_{2}$ increases from 0 to a small positive number, chaos changes to limit cycle while the equilibrium $\mathbf{u}^{(\mathbf{9})}$ is still unstable. For example, when $b_{2}=0.01$, the equilibrium $\mathbf{u}^{(\mathbf{9})}=$ $[33.9297,13.3333,17.4531]$ is still unstable. However, when $b_{2}$ increases a little bit, the equilibrium $\mathbf{u}^{(\mathbf{9})}$ becomes stable and the solutions will converge to it. For example the equilibrium $\mathbf{u}^{(\mathbf{9})}=[33.9297,13.3333,6.1399]$ becomes stable and the solutions will converge to it.

In summary, the intra-species competition among specialist predator $b_{2}$ can stablizes the system by making the unstable equilibrium $\mathbf{u}^{(\mathbf{9})}$ stable. With the help of intra-species competition, large fluctuations of the population distribution such as limit cycle or chaos can be reduced and they can become stable steady states. 


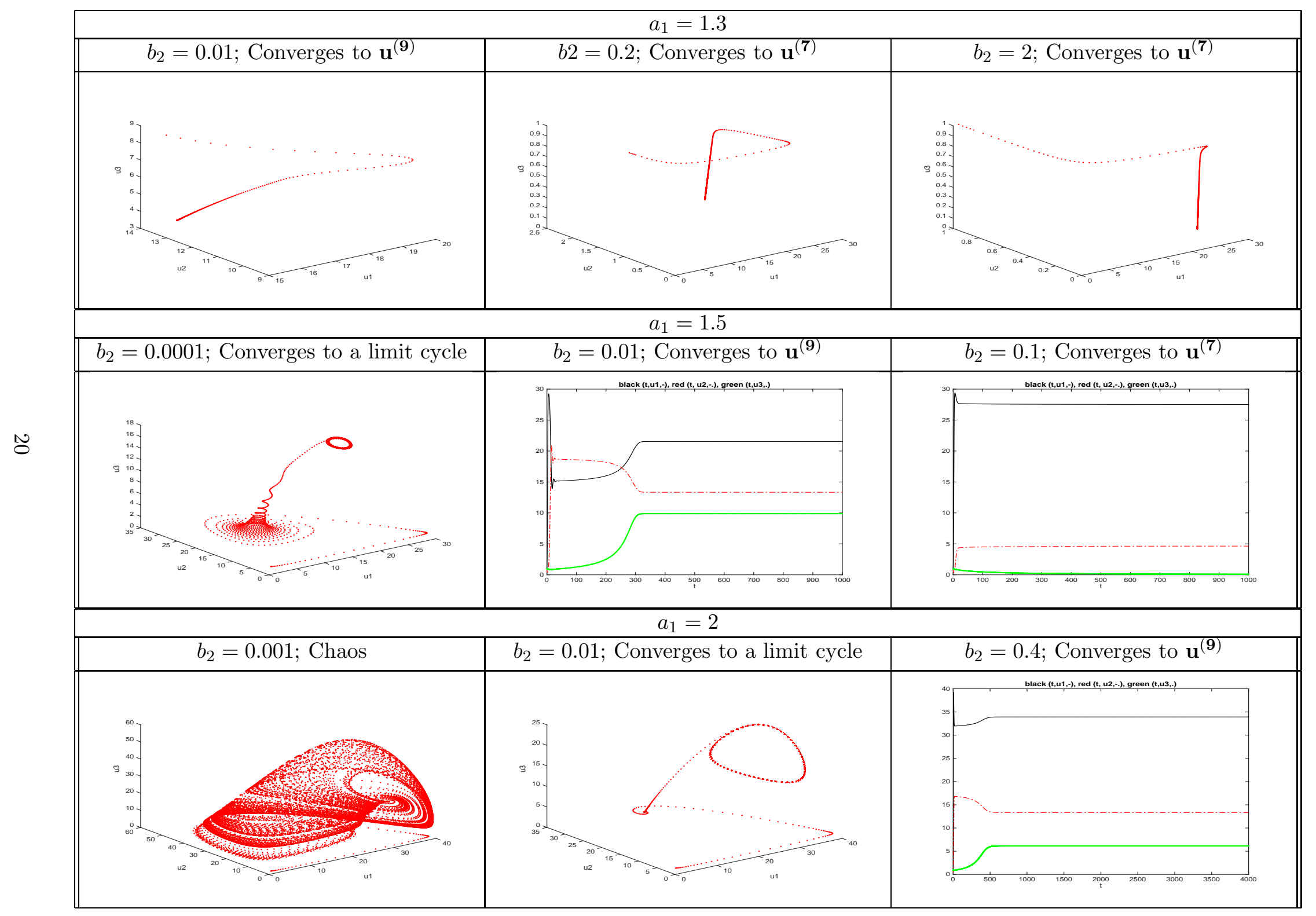

Table 2: The values of other parameters are given in (21) and (22). Graphs are either solution curves over time or phase portrait. 


\subsection{Turing instability induced by cross diffusion and Turing patterns}

In the above subsections, we fixed the parameters in (21) and (22) and showed that the reproduction rate $a_{1}$ and the intra-species competition $b_{2}$ can induce the stable coexistence equilibrium, partial extinction, limit cycle, and chaos in the ODE system. In this subsection, using numerical methods, we illustrate that the cross-diffusion induce spatial patterns.

Throughout this subsection we assume that the region of the PDE system (2) is a fixed rectangular domain $\Omega=[0, L] \times[0, L] \subset \mathbf{R}^{2}$. We modify the numerical method in [15] to solve the PDE system (2). We found the numerical solution of the PDE system on a grid with $100 L \times 100 L$ nodes using a simple standard Euler method with a time step of $\triangle t$.

We discretize the Laplacian in the grid with lattice sites denoted by $(i, j)$. The form is

$$
\begin{aligned}
\left.\triangle w\right|_{(i, j)}= & \frac{1}{s^{2}}\left(a_{l}(i, j) w(i-1, j)+a_{r}(i, j) w(i+1, j)\right. \\
& +a_{d}(i, j) w(i, j-1)+a_{u}(i, j) w(i, j+1)-4 w(i, j),
\end{aligned}
$$

where $s$ is the lattice constant and the matrix elements of $a_{l}, a_{r}, a_{d}, a_{u}$ are unity except at the boundary. When $(i, j)$ is at the left boundary, that is $i=0$, in order to have zero-flux of reactants in the left boundary, we define

$$
a_{l}(i, j) w(i-1, j) \equiv w(i+1, j) .
$$

Similarly, we define $a_{r}(i, j), a_{d}(i, j), a_{u}(i, j)$ such that the boundary is no flux. Notice that approximation formula for the Laplacian operator (23) can be used to approximate Laplacian $u_{1}, u_{2}$, and $k_{12} u_{1} u_{2}$ etc.

Parshad, Kumari, Kasimov, and Abderrahmane [21] conducted numerical simulations to explore the spatiotemporal dynamics in two dimensional spatial domain. Some nice Turing patterns such as spots, or a mix of spot and labyrinth were numerically simulated. To compare our results with theirs, we set some parameters the same as the one used in [21]. We choose a rectangular domain [3.14,3.14] with a grid of $\triangle x=\triangle y=0.01$ and a time step of $\triangle t=0.001$. The common parameters are taken as

$$
\begin{aligned}
& a_{1}=2 ; a_{2}=0.8 ; a_{3}=0.04 ; b_{1}=0.15 ; w_{0}=0.55 ; w_{1}=2 \\
& w_{2}=.5 ; w_{3}=1.2 ; D_{0}=10 ; D_{1}=13 ; D_{2}=10 ; D_{3}=20 .
\end{aligned}
$$

We first reconstruct the spot pattern (Fig. 5 in [21]) by using the following parameters

$$
b_{2}=0 ; k_{11}=0.01 ; k_{22}=0.00001 ; k_{33}=0.01 ; k_{i j}=0 \text { for } i \neq j
$$

The initial distribution of the species is considered to be a small spatial perturbation of the form $0.1 \cos ^{2}(10 x) \cos ^{2}(10 y)$ about the coexistence steady state. The result of the simulation is presented as contour plot in Figure 4 and as surface plot in Figure 5. The pattern may also depend on the initial distribution of the species. If the initial distribution of the species is taken as a small perturbation of the form $0.1 \cos ^{2}(10 x)$, the result of the simulation shows the strip patterns in Figure 6 as contour plot and in Figure 7 as surface plot. 
If $b_{2}=0$ is replaced by $b_{2}=0.005$ in $(25)$, the equilibrium $\mathbf{u}^{(9)}$ is linearly stable when $k_{i j}=0$ for $i \neq j$ by theorem 3.1. No new pattern is generated and the solutions are convergent to homogenous equilibrium solution in our numerical simulations. In order to have new patterns, the parameter $k_{i j}$ should not be all zeros.

$$
\begin{aligned}
& b_{2}=0.005 ; k_{11}=0.01 ; k_{22}=0.00001 ; k_{33}=0.01 \\
& k_{21}=0.0001 ; k_{23}=0 ; k_{23}=0 ; k_{32}=0.0039 .
\end{aligned}
$$

When the parameters in (25) are replaced by (26), the coexistence equilibrium $\mathbf{u}^{(9)}$ is $(11.6389,10,3.7902)$ and it is linearly stable for the ODE system (1) by theorem 2.1 . Conditions (7) in theorem 2.1 and conditions (19) in theorem 4.1 are both satisfied. By theorem 4.1, Turing instability may occur when cross-diffusion $k_{21}$ or $k_{32}$ are not zero and are larger than certain values. New pattern is found numerically (see Figure 8 and Figure 9 ) and it is very different from the patterns shown in Pashad's paper [21]. The stable steady state $\mathbf{u}^{(9)}$ is destabilized by cross-diffusion. But the new patten is not a stable steady spatial pattern and it is almost periodically fluctuation like a wave over time. In order to show the differences of the patterns, Figure 10 presents three solution curves starting at the same space point $(1,1)$ over a long time period. The convergence of top row graphs in Figure 10 implies that the spot patten (Figure 4) and strip pattern (Figure 6) are steady patterns. But the almost periodically varying pattern in the lower row graph in Figure 10 show that the pattern in Figure 8 keeps periodically changing. If the parameter $k_{32}$ takes smaller value while not changing the other parameters in (24) and (26), spatial solutions converge to flat equilibrium. If the parameter $k_{32}$ takes larger value, spatial solutions may not exist any more and they become unbounded.

\section{Conclusion and Discussion}

In this paper, we have studied analytically as well as numerically a coupled three-species food chain model (2). We extend the results in paper [21] by introducing the intra-species competition $b_{2}$ and cross-diffusions. The model of the ODE system (1) exhibits very rich dynamics that include stable steady states, limit cycles, and chaos (see table 1 and 2). The main goal of the paper is to study the spatial pattern formation of the system (2) . We have shown that classical Turing instability induced by self-diffusion does not occur for a stable region when conditions (7) are satisfied. But under some conditions, the model generates spatial patterns only in the presence of cross-diffusion. The phenomenon can be regarded as the extension of Turing patterns and it is called Turing instability induced by cross-diffusion. Moreover, numerical simulation of Turing patterns in two dimension is given to illustrate some spatial patterns such as the spot pattern (Figure 4 and 5), strip pattern (Figure 6 and 7), and almost periodically fluctuation pattern. Hence, we have illustrated numerically the existence of nonuniform steady state solutions. 

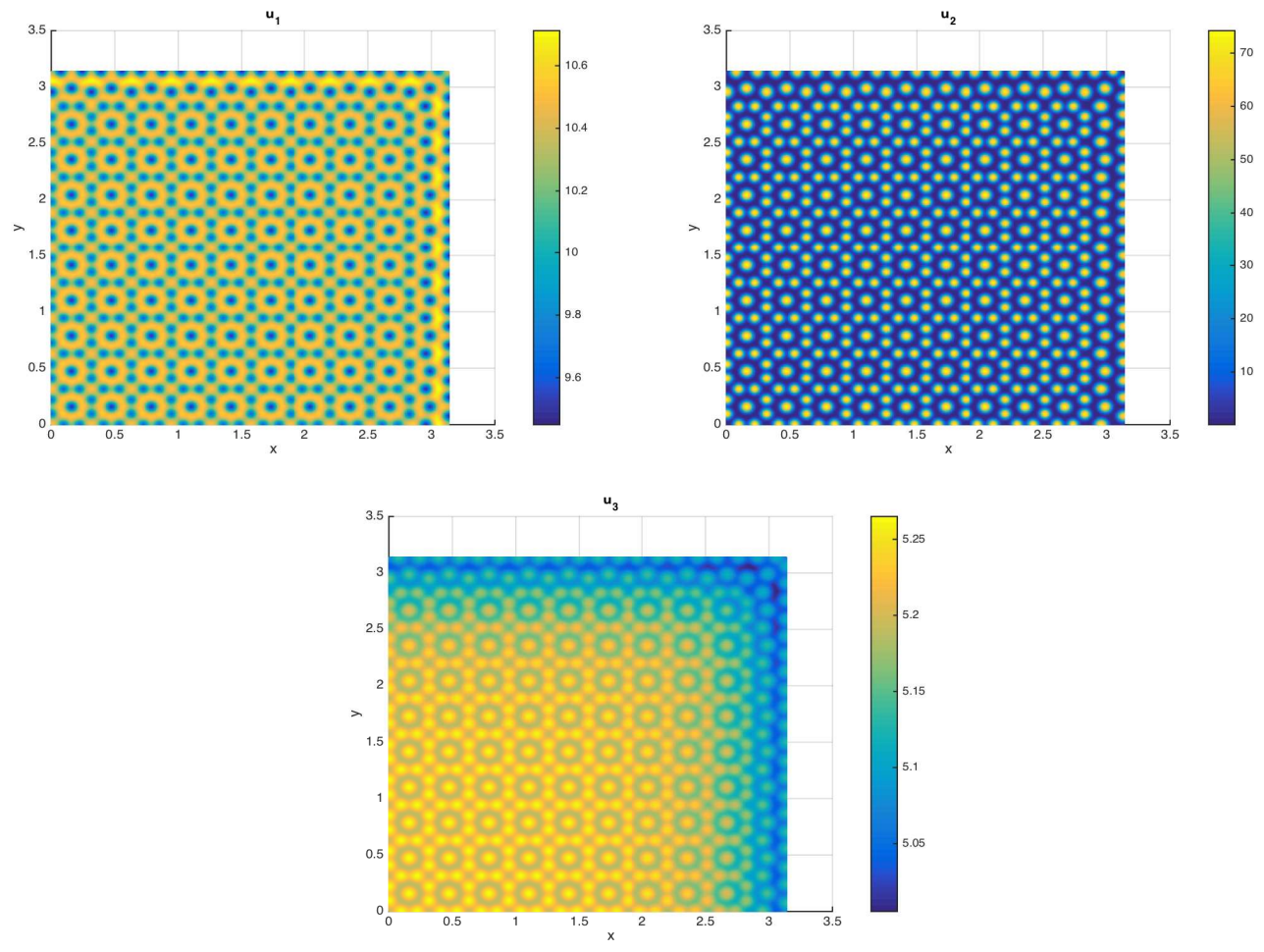

Figure 4: The densities of the three species are shown as contour plots in the $x y$-plane. Parameters are given in (24) and (25). The longterm is seen to be spot Turing patterns which have been demonstrated in the paper [21]. 

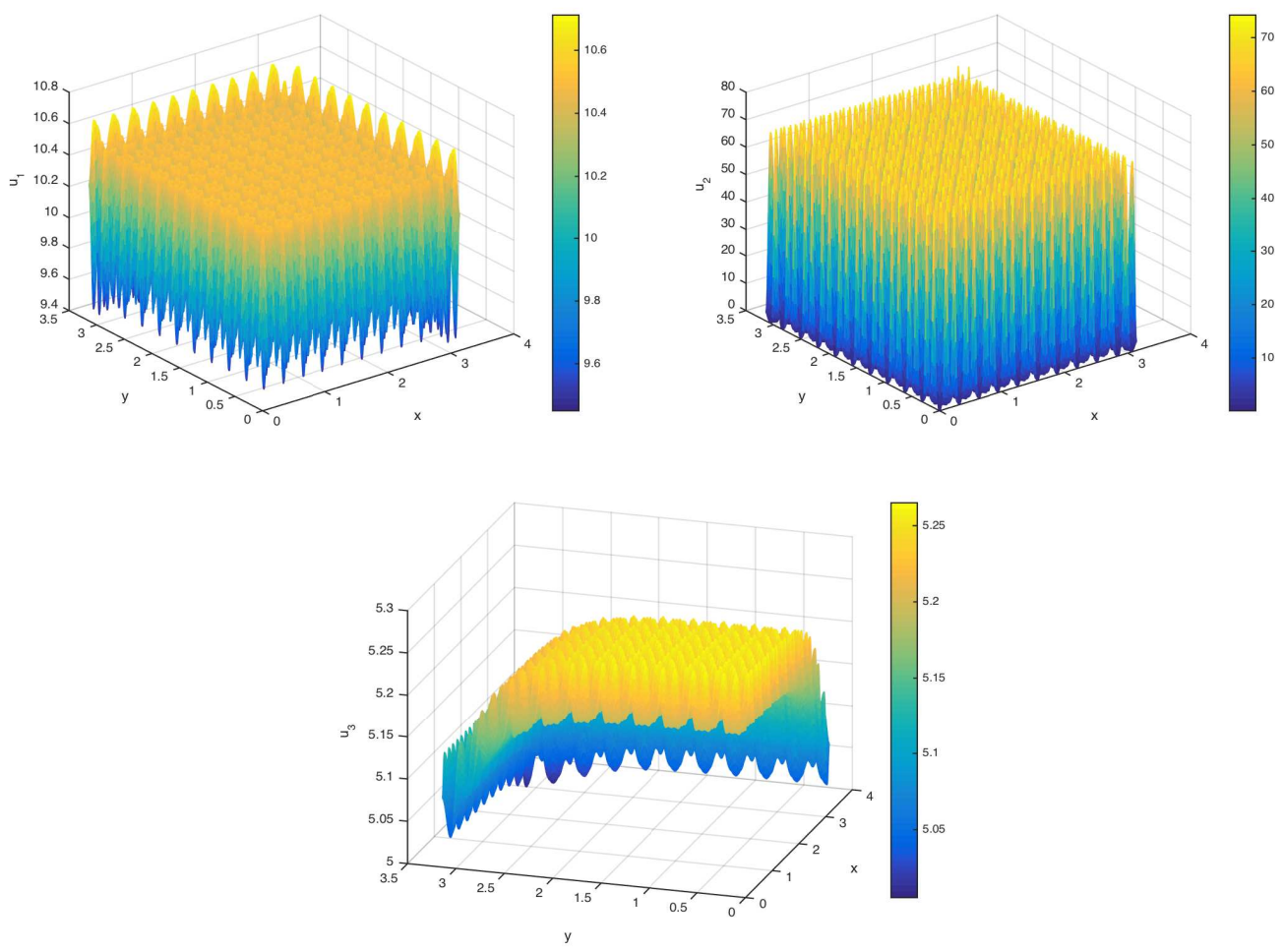

Figure 5: Spatial distributions of the species as surfaces are shown, matching with the contour plot of Figure 4 . 

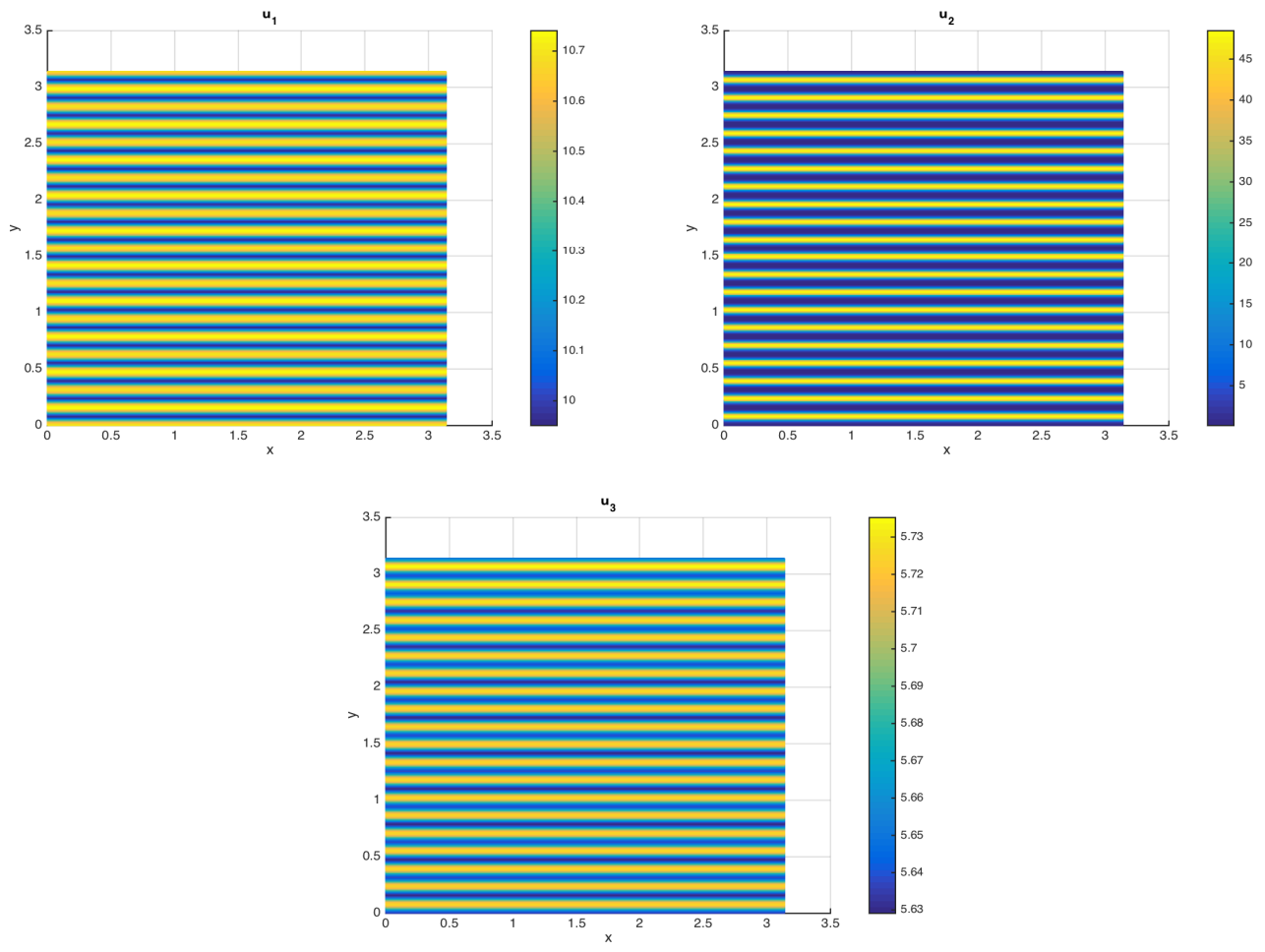

Figure 6: Spatial distributions of the species as contour plot are shown with parameters given in (24) and (25). Strip patterns are presented and they are not appeared in reference [21]. 

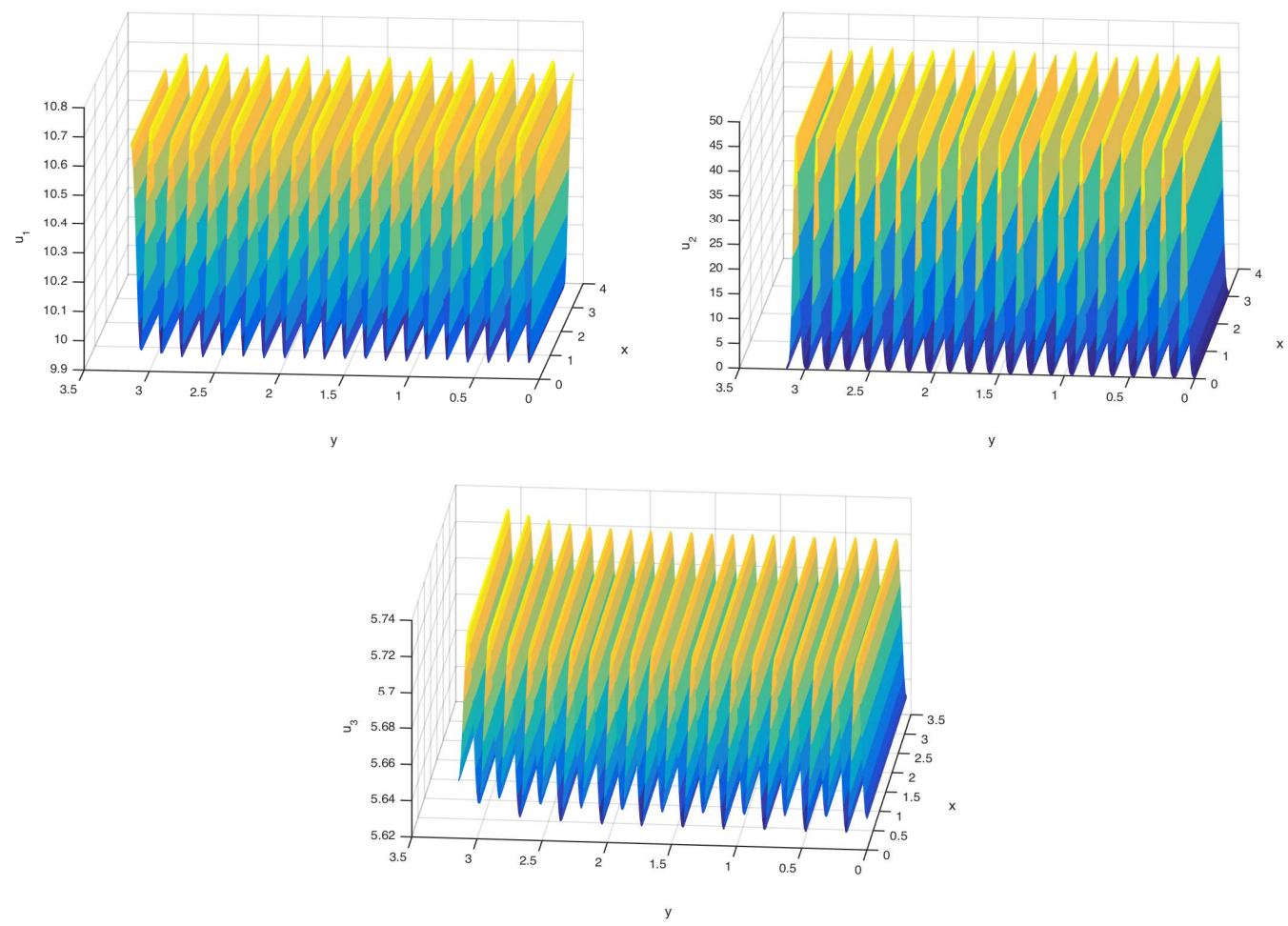

Figure 7: Spatial distributions of the species as surfaces are shown with parameters given in (24) and (25), matching with the contour plot of Figure 6 . 

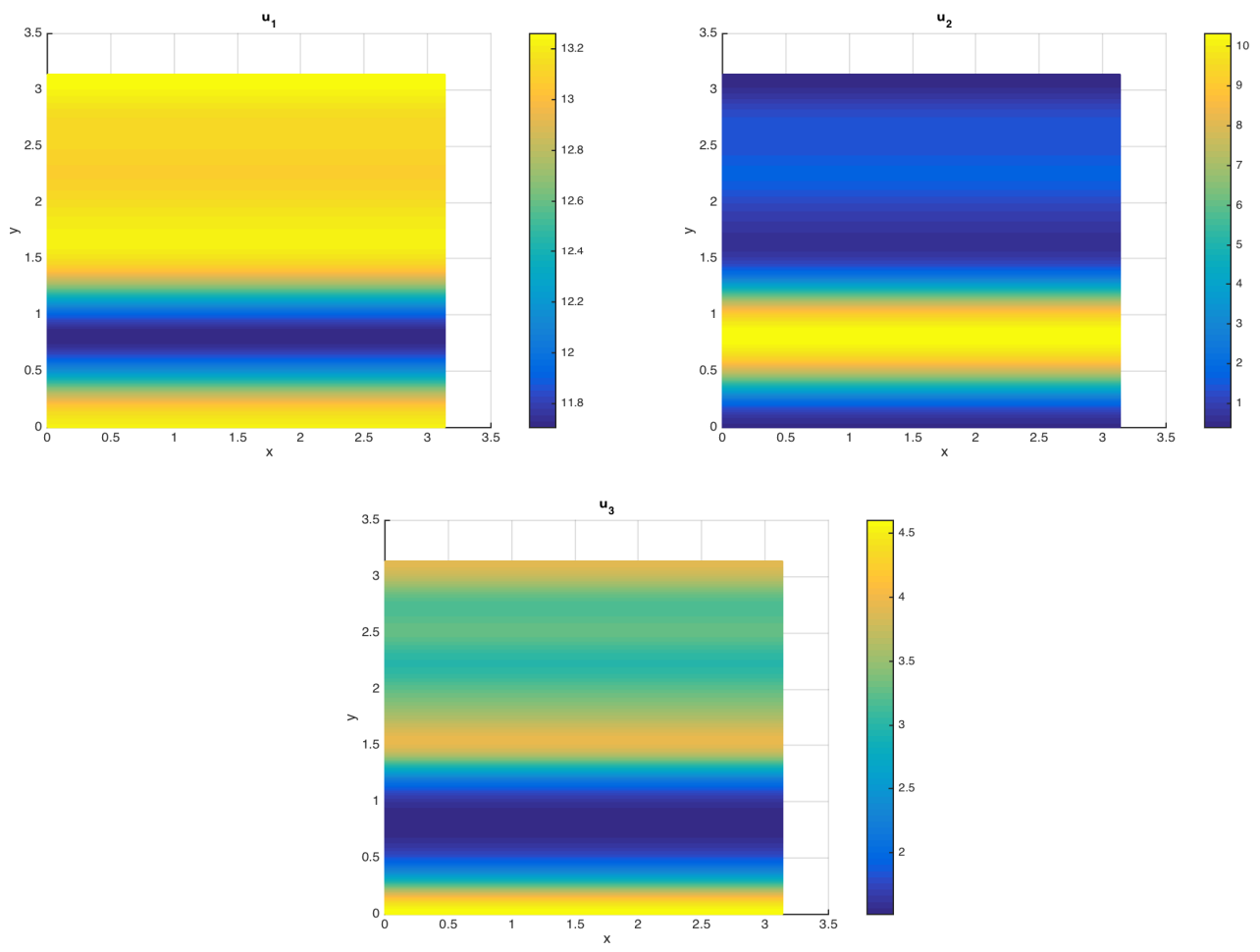

Figure 8: Spatial distributions of the species as contour plot are shown for parameters given in (24) and (26). This is different from the steady spot patterns in Fig. 4 and strip patterns in Fig. 6. It is not a steady state pattern and spatial distributions are almost periodically fluctuated over time. 

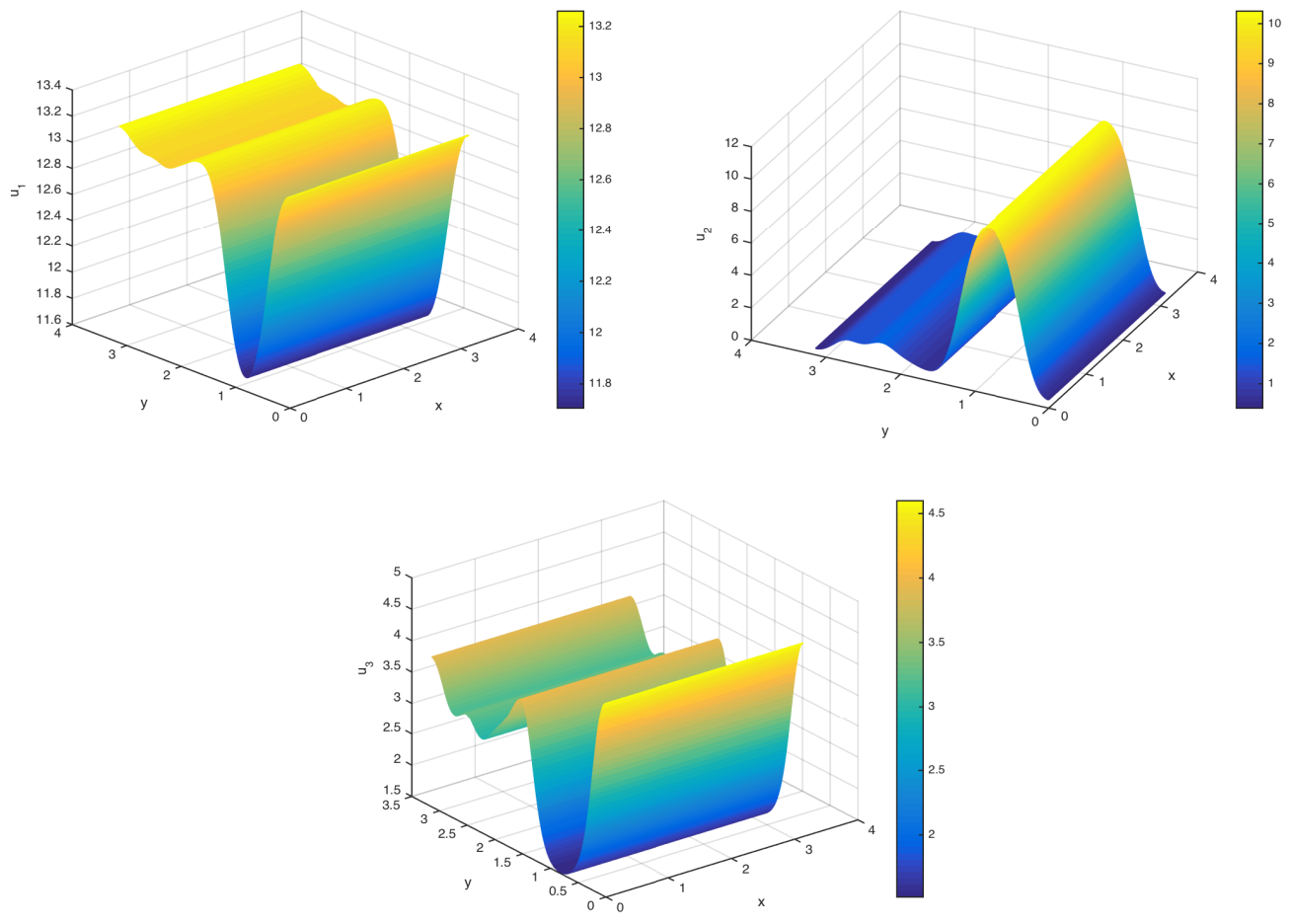

Figure 9: Spatial distributions of the species as surfaces are shown with parameters are given in (24) and (26), matching the contour plot 8. 

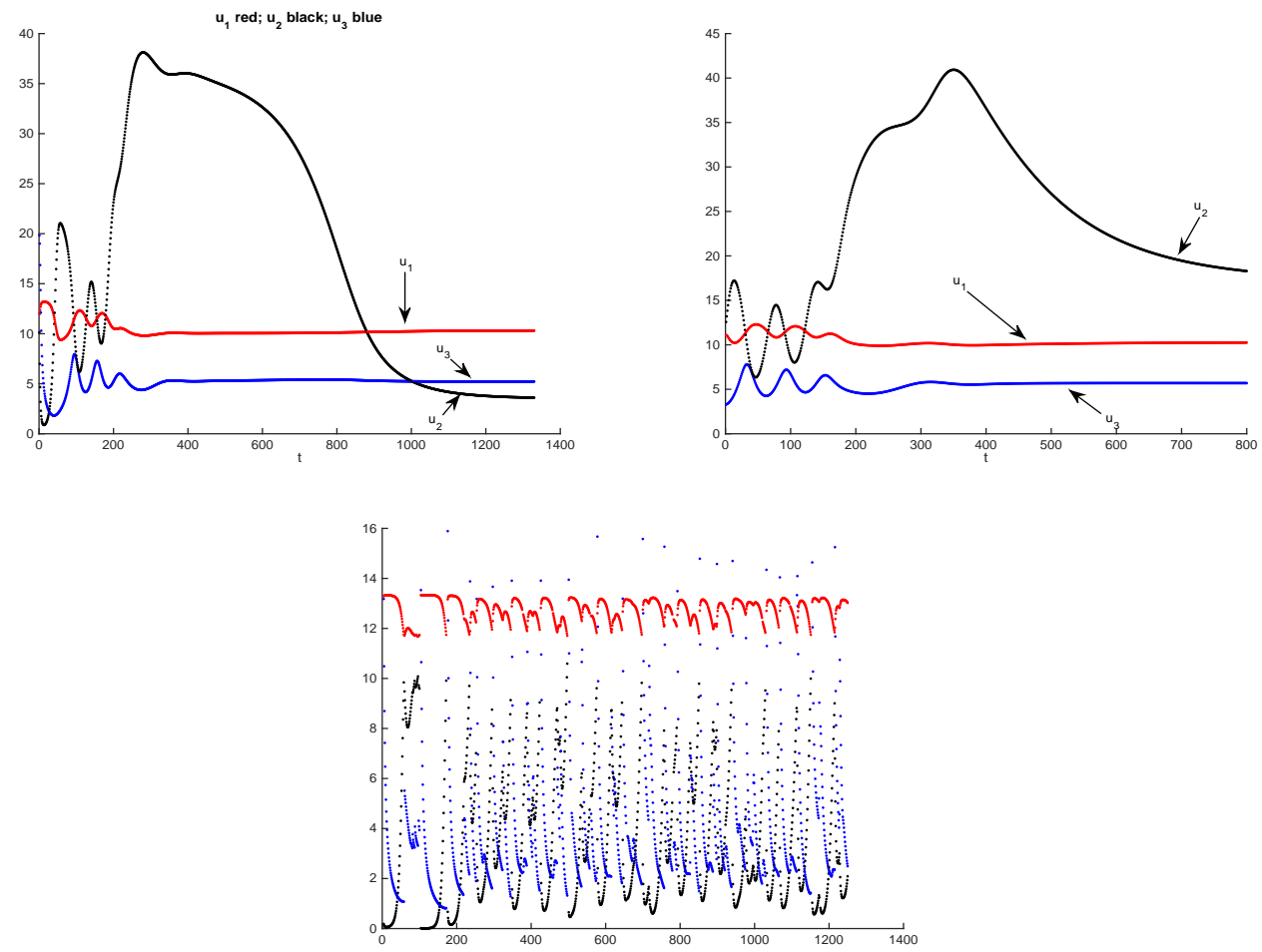

Figure 10: The curves in these graphs are the density function $u_{i}(t)$ starting from a same space location over time $t$. The convergence of the curves in the top two graphs shows that the spot pattern (Figure 4) and strip pattern (Figure 7) are steady state. But the periodically changing of the curves in the lower graph shows that the pattern in Figure 9 is not a steady state and the spatial distributions are fluctuated periodically over time. 


\section{Acknowledgements}

The authors would like to thank the editors and the anonymous referees for their valuable comments and suggestions regarding this work. Zhifu Xie also gratefully acknowledges the support from NSF grant HRD-1409939.

\section{References}

[1] M.A. Aziz-Alaoui, Study of a Leslie-Gower type tri-trophic population model, Chaos, Solitons \& Fractals, 14 (8), (2002), 1275-1293.

[2] X. Chen, Y. Qi, M. Wang, A strongly coupled predator-prey system with nonmonotonic functional response, Nonl. Anal.: TMA 67 (2007) 1966-1979.

[3] Y.H. Du, J.P. Shi, Some recent results on diffusive predator-prey models in spatially heterogeneous environment, in: Nonlinear Dynamics and Evolution Equations, in: Fields Inst. Commun., Vol. 48, Amer. Math. Soc., Providence, RI, 2006, 95-135.

[4] Y.H. Du, J.P. Shi, A diffusive predator-prey model with a protection zone, J. Differential Equations 229 (1) (2006) 63-91.

[5] A. Hastings, T. Powell, Chaos in a three species food chain, Ecology, 72 (1991), 896903.

[6] K. Kim, Z. Lin, Coexistence of three species in a strongly coupled elliptic system, Nonl. Anal. 55 (2003) 313-333.

[7] K. Kim, Z. Lin, Blow up in a three species cooperating model, Appl. Math. Let. 17 (2004) 89.

[8] N. Kumari, Pattern formation in spatially extended tritrophic food chain model system: Generalist versus Specialist top predator, ISRN Biomathematics Vol. 2013, Article ID 198185, 12 pages.

[9] J. D. Murray, Mathematical biology. Third edition. I. An introduction. Interdisciplinary Applied Mathematics, 17. Springer-Verlag, New York, 2002; II. Spatial models and biomedical applications. Interdisciplinary Applied Mathematics, 18. Springer-Verlag, New York, 2003.

[10] A. Okubo, Diffusion and Ecological Problems: Mathematical Models, Springer-Verlag, Berlin, 1980.

[11] P.Y.H. Pang, M. Wang, Strategy and stationary pattern in a three-species predatorprey model, J. Differential Equations 200 (2004) 245-273.

[12] W. H. Ruan, Positive steady-state solutions of a competing reaction-diffusion system with large cross-diffusion. J. Math. Anal. Appl. 197(1996) 558-578. 
[13] J. Shi, Z. Xie and K. Little, Cross-diffusion induced instability and stability in reactiondiffusion systems, Journal of Applied Analysis and Computation, Vol. 1, No. 1 (2011) pp 95-119.

[14] A.M. Turing, The chemical basis of morphogenesis. Phil. Trans. Royal Soc. London. B237, (1952), 37-72.

[15] C. Tian, Turing patterns created by cross-diffusion for a Holling II and Leslie-Gower type three species food chain model, J. Math. Chem. (2011) 49: 1128-1150.

[16] M. Wang, Stationary patterns of strongly coupled prey-predator models, J. Math. Anal. Appl. 292 (2004) 484-505.

[17] R.K. Upadhyay, S.R.K. Iyengar, and V. Rai, Chaos: An ecological reality?, International Journal of Bifurcations and Chaos, 8 (1998) 1325-1333.

[18] R. K. Upadhyay, S.R.K. Iyengar, and V. Rai, Why chaos is rarely observed in natural populations? Chaos, Solitons and Fractals, 8(12), (1997), 1933-1939.

[19] R.D. Parshad, R. K. Upadhyay, Investigation of long time dynamics of a diffusive three species aquatic model, Dynamics of Partial Differential Equations, 7(3), (2010),217244.

[20] R.D. Parshad, H. Abderrahmanne, R.K. Upadhyay, and N. Kumari, Finite time blowup in a realistic food chain model, International Scholarly Research Notices, Volume 2013 (2013), Article ID 424062.

[21] R.D. Parshad, N. Kumari, A.R. Kasimov, H.A. Abderrahmane, Turing patterns and long-time behavior in a three-species food-chain model, Mathematical Biosciences 254 (2014) 83-102.

[22] R.D. Parshad, N. Kumari, and S. Kouachi, A remark on "Study of a Leslie-Gowertype tritrophic population model [Chaos, Solitons and Fractals 14 (2002) 1275-1293]", Chaos, Soli- tons \& Fractals, 71(2), 22-28, 2015.

[23] Z.F. Xie, Turing instability in a coupled predator-prey model with different Holling type functional responses, Discrete and Continuous Dynamical System, Series S, 4 (2011) pp 1621-1628.

[24] Z.F. Xie, Cross-diffusion induced Turing instability for a three species food chain model, Journal of Mathematical Analysis and Applications, 388(1), (2012), 539-547.

[25] F. Yang, S. Fu, Global solutions for a ti-trophic food chain model with diffusion, Rocky Mt. J. Math. 38 (5) (2008) 1785.

[26] X. Zeng, Non-constant positive steady states of a prey-predator system with crossdiffusions, J. Math. Anal. Appl. 332(2007) 989-1009. 
[27] J. Zhou, C. Kim, J. Shi, Positive steady state solutions of a diffusive Leslie-Gower predator-prey model with Holling type II functional response and cross-diffusion. Discrete Contin. Dyn. Syst. 34 (2014), no. 9, 3875-3899. 